BNL-76747-2006

FORMAL REPORT

\title{
Parton Bubble Model for Two Particle Angular Correlations at RHIC/LHC
}

\author{
S.J. Lindenbaum ${ }^{a, b}$ and R.S. Longacre ${ }^{a}$ \\ ${ }^{a}$ Brookhaven National Laboratory, Upton, NY 11973 USA \\ ${ }^{b}$ City College of New York, NY 10031 USA
}

June 2006

Physics Department/STAR Group

\author{
Brookhaven National Laboratory \\ P.O. Box 5000 \\ Upton, NY 11973-5000 \\ www.bnl.gov
}

Notice: This manuscript has been authored by employees of Brookhaven Science Associates, LLC under Contract No. DE-AC02-98CH10886 with the U.S. Department of Energy. The publisher by accepting the manuscript for publication acknowledges that the United States Government retains a non-exciusive, paid-up, irrevocable, world-wide license to publish or reproduce the published form of this manuscript, or allow others to do so, for United States Government purposes. 


\section{DISCLAIMER}

This report was prepared as an account of work sponsored by an agency of the United States Government. Neither the United States Government nor any agency thereof, nor any of their employees, nor any of their contractors, subcontractors, or their employees, makes any warranty, express or implied, or assumes any legal liability or responsibility for the accuracy, completeness, or any. third party's use or the results of such use of any information, apparatus, product, or process disclosed, or represents that its use would not infringe privately owned rights. Reference herein to any specific commercial product, process, or service by trade name, trademark, manufacturer, or otherwise, does not necessarily constitute or imply its endorsement, recommendation, or favoring by the United States Government or any agency thereof or its contractors or subcontractors. The views and opinions of authors expressed herein do not necessarily state or reflect those of the United States Government or any agency thereof. 


\title{
Parton Bubble Model for Two Particle Angular Correlations at RHIC/LHC S.J. Lindenbaum ${ }^{a, b}$, R.S. Longacre ${ }^{a}$ ${ }^{a}$ Brookhaven National Laboratory, Upton, NY 11973, USA ${ }^{b}$ City College of New York, NY 10031, USA ${ }^{1}$
}

\begin{abstract}
In an earlier paper we developed a bubble model, based on a view we had shared with van Hove for over two decades. Namely, that if a quark-gluon plasma is produced in a high energy heavy ion collider, then its hadronization products would likely be emitted from small bubbles localized in phase space containing plasma. In this paper we refined the model to become a parton bubble model in which each localized bubble contains initially 3-4 partons which are almost entirely gluons forming a gluon hot spot. We greatly expanded the transverse momentum interval investigated, and thus are able to treat recombination effects within each bubble. We again utilize two particle correlations as a sensitive method for detecting the average bubble substructure. In this manuscript we make many predictions for angular correlations detectable at RHIC and which will be later modified to LHC conditions. Some early available low precision correlation analyses is qualitatively explained. However a critical consistency test of the model can be made with high precision data expected in the near future.
\end{abstract}

\section{Introduction and General considerations}

In an earlier publication[1] we introduced a bubble model. In this paper we further developed the ideas we have shared with van Hove for over two decades refs.[2-4]. Namely we anticipated, that in heavy ion collisions at energies that are accessible at colliders such as RHIC or LHC, if a quark-gluon plasma(QGP) is created it is more probable to produce bubbles (or droplets) containing QGP localized in phase space. In ref.[1] we proposed a bubble model for high energy heavy ion collisions, consisting of a single ring of about a dozen adjoining $2 \mathrm{fm}$ radius bubbles transverse to the collider beam direction, centered on the beam, and located at or near mid-rapidity(see Figure 1). This is the geometry for the final state kinetic freezeout of the QGP bubbles on the surface of the expanding fireball. In the central (near impact parameter 0 ) mid-rapidity region at $\mathrm{RHIC}$ we are observing the region where the highest energy densities and temperatures (parton energies) are produced. The $\sqrt{s_{N N}}=200$ $\mathrm{GeV}$ central $\mathrm{Au}+\mathrm{Au}$ collisions at RHIC produce initial energy densities[5] which exceed those predicted by lattice quantum chromodynamics (QCD) as sufficient for production of a QGP[6].

We consider it most likely that evidence for a bubble substructure (dense gluonic hot spots) perhaps originating from a quark gluon plasma (QGP) could be found in particle correlations produced in these collisions.

\footnotetext{
${ }^{1}$ This research was supported by the U.S. Department of Energy under Contract No. DE-AC0298CH10886 and the City College of New York Physics Department
} 
Plane section of bubble geometry perpendicular to the beam at eta $=0$

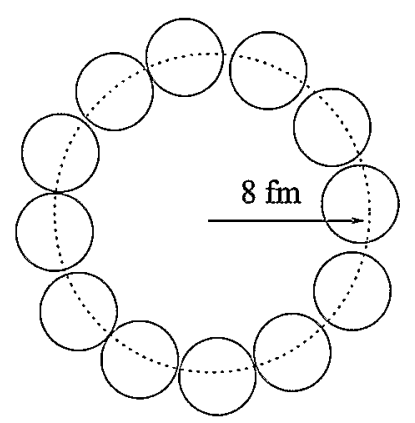

Projection of the bubble geometry on a plane containing the beam line

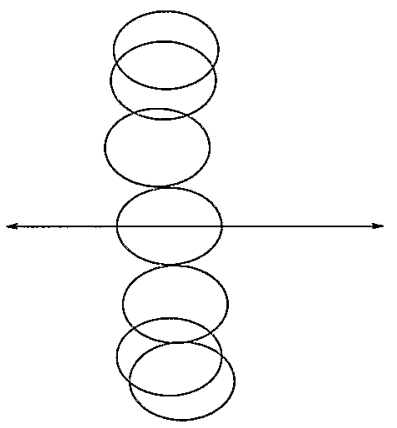

A perspective view of the bubble geometry.

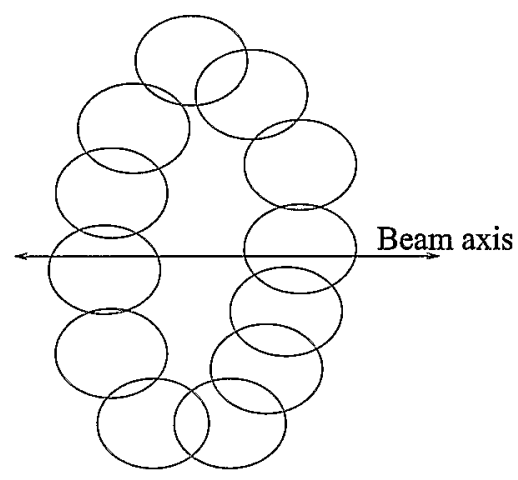

Figure 1: The bubble geometry is an $8 \mathrm{fm}$ radius ring composed of twelve adjacent $2 \mathrm{fm}$ radius spherical bubbles elongated along the beam direction by the Landau longitudinal expansion. The upper left figure is a plane section perpendicular to the beam at $\eta=0$. The lower left fiqure is a projection of the bubble geometry on a plane containing the beam line. The lower right figure is a perspective view of the bubble geometry. 
The bubble ring radius of our model was estimated by blast wave and other general considerations to be approximately $8 \mathrm{fm}$. This single bubble ring resides on the surface of the expanding fireball at freezeout. Each bubble would emit a considerable fraction of final state particles resulting from the QGP state and there would be very little re-interaction for those emitted outward from the surface because the surface of the fireball is at freezeout. The choice of the $2 \mathrm{fm}$ radius was motivated by the Hanbury-Brown and Twiss (HBT) [7] observations which have shown that at low $p_{t}$ the final state source size radius is about $6 \mathrm{fm}$, whereas above $0.8 \mathrm{GeV} / \mathrm{c}$ the source size reduced to about a $2 \mathrm{fm}$ radius.

The $2 \mathrm{fm}$ source size is interpreted as phase space focusing of the viewed region of the overall source becoming smaller and near the surface as one selects higher than $0.8 \mathrm{GeV} / \mathrm{c}$ $p_{t}$ particles. Phase space focusing makes it possible for these higher $p_{t}$ particles to view and resolve individual $2 \mathrm{fm}$ radius regions(bubbles) on the surface of the expanding fireball. If there is such a bubble substructure it would appear as angular regions of greater particle production above a $p_{t}$ momentum of $0.8 \mathrm{GeV} / \mathrm{c}$. Therefore two-particle angular correlations could detect this bubble substructure. The difference in angles particle correlation techniques we are employing are powerful methods in determining bubble substructure since it is the relative angles of the two-particles not their position on the fireball surface that is important. Thus our bubble ring of approximately $2 \mathrm{fm}$ radius bubbles would lead to the phase space focusing which produced the approximately $2 \mathrm{fm}$ final state source size obseved by HBT[7] since the 12 bubbles would image on top of each other.

In ref.[1] we took account of the well known Landau longitudinal expansion of the fireball that causes the angular spread of particles to be much larger in the longitudinal direction than the transverse. Experimental progress at RHIC leads us to speculate that high precision experimental data publications in the near future are likely to appear that can test and are compatible with our model.

In this paper we expand the model of ref.[1] and employ a 12 bubble ring similar to the previous work, but treat mid-rapidity $\mathrm{Au}+\mathrm{Au} \sqrt{s_{N N}}=200 \mathrm{GeV}$ central production (impact parameter near 0). Using RHIC Au + Au data with $\sqrt{s_{N N}}=200 \mathrm{GeV}$, we refine our model and make predictions for angular correlation analyses of charged particles emitted from the surface of the fireball.

We will concentrate on the experimentally observed particle pair angular correlations in the transverse momentum region $0.8 \mathrm{GeV} / \mathrm{c}<p_{t}<4.0 \mathrm{GeV} / \mathrm{c}$, except those at small relative angles where Coulomb and Hanbury-Brown Twiss(HBT) effects occur. Background resonances are also considered unimportant. Figure 1 shows a sketch of the bubble geometry used. Our goal is to develop a parton inspired bubble model. Thus we assume that essentially all mid rapidity two particle angular correlations in the final state of central heavy ion collisions, except the small angle Coulomb and HBT effects, results from particle emission by the parton bubbles formed on the outer surface of the expanding fireball. The present paper will concern itself with predictions that are testable at RHIC. However when sufficient knowledge of the characteristics of future LHC data becomes available we intend to apply these ideas, suitably modified for conditions at LHC, to that data.

In our earlier work[1], we made a cut on transverse momentum $0.8 \mathrm{GeV} / \mathrm{c}<p_{t}<2.0$ 
$\mathrm{GeV} / \mathrm{c}$. The lower bound was chosen to resolve individual bubbles and take advantage of phase space focusing to enhance the bubble signal, by adding up contributions from many bubbles. The upper bound was chosen in order to avoid possible jet fragmentation contamination. However now it is clear that jets are strongly quenched[8], and provided one does not use a jet trigger the particles in the 2.0 to $4.0 \mathrm{GeV} / \mathrm{c} p_{t}$ range are not the usually expected jet fragmentation particles. For example many of the particles exhibit $\eta$ elongation very inconsistent with jet characteristics[9]. In the present work we think it is reasonable to assume that a large fraction of the particle correlations in the 0.8 to $4.0 \mathrm{GeV} / \mathrm{c}$ $p_{t}$ range come from bubbles. Expanding our upper cut to $4.0 \mathrm{GeV} / \mathrm{c}$ allows us to explore quark-quark recombination[10] and various combinations of 2-particle correlations such as for example angular correlations of protons and anti-protons with other charged particles. The kinematical consequences of quark-quark recombination require the consideration of this higher $p_{t}$ cutoff. Furthermore it appears reasonable to make the approximation that for central $\mathrm{Au}+\mathrm{Au}$ collisions at RHIC, jet fragmentations from parton - parton scattering are small compared to our bubble fragmentation, because of jet quenching. Parton - parton scattering is an initial state effect which is reduced by the medium, whereas our bubbles are the final state source on the outer surface of the fireball.

\section{Our Parton Bubble Model}

In our earlier work[1], we constructed a model for bubble formation based on the modification of the HIJING event generator[11]. The most important modification was the replacement of the jets ( sometimes referred to as mini-jets) with bubbles. We also modified the particles from the remaining beam jets fragmentation by including the effects of elliptic flow. For this work HIJING is further modified so as to merely become a source of background particles. HIJING has two relevant sources of particle production: Jets which fragment into particles which are referred to as jet particles, and the soft particles which come from beam jet fragmentation. The jet particles are not flat in azimuth but bunch around the jet axis. The beam jets fragmentations are very flat in azimuth.

Also in this work we make the approximation that jet particles are essentially removed or have their correlations eliminated by quenching, and thus there are no correlations left due to jets. Only the soft beam jet particles from HIJING are left and they do not have any correlations. Therefore, they are our background particles in the correlation investigations. However, we did include the effects of elliptic flow[12] on the soft beam jet particles. Momentum, energy, and charge conservation are all satisfied within the bubble ring in this model.

A parton that forms a jet fragments into particles through a QCD shower process which finally forms quarks and anti-quarks in colored pairs. These quarks and anti-quarks combine into color singlet hadrons which form the jet particles. The spectrum of initial jet partons is given by the saturation scale and perturbative quantum chromodynamics (pQCD) cross sections. The parton scattering occurs as binary scattering of two partons leading to a dijet structure. The dijets to lowest order scatter in opposite azimuthal directions (back to back), 


\section{Jet}

\section{Parton Shower}

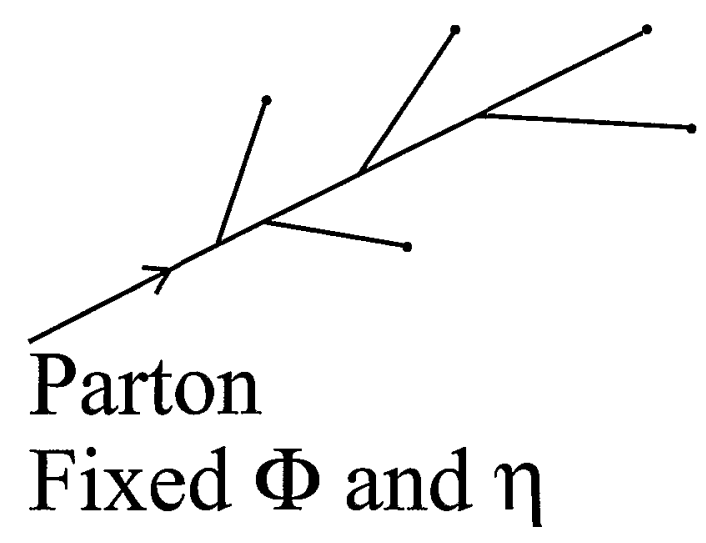

Figure 2: A jet parton shower.

while the amount of longitudinal momentum varies. Each jet has a well defined $\eta$ and $\Phi$ direction(see Figure 2).

Particle production from our bubbles uses a similar parton QCD shower fragmentation as a jet with a well defined $\Phi$ angle. Inside the bubble there are 3-4 partons with differing longitudinal momentum. The $p_{t}$ distribution of the partons inside the bubble is similar to pQCD but has a suppression in the high $p_{t}$ region like the data[8]. We use Pythia fragmentation functions[13] for the bubble fragmentation. The experimentally tuneable adjustments in the model are the number of partons in a bubble and the longitudinal momentum of each parton which can be different ( see Figure 3).

In order to tune these adjustments we utilize some central RHIC data. The STAR experiment has measured charged particle pair correlations for approximately $0-10 \%$ centrality $\mathrm{Au}+\mathrm{Au}$ events at $\sqrt{s_{N N}}=200 \mathrm{GeV}$ [14]. The $p_{t}$ range of that data is 0.8 to $2.0 \mathrm{GeV} / \mathrm{c}$ for the $\eta$ range $|\eta|<1.0$. Two $\Delta \eta$ bins are presented in that analysis $(0.0<\Delta \eta<0.3$ and $0.6<\Delta \eta<0.9)$. For each simulated event we added the particles emitted from 12 bubbles and enough soft beam jets in order to have an average of 186 charged particles per event in order to agree with the data. The tunable parameters in our parton bubble model are the number of partons per bubble and their longitudinal distribution. 200,000 events 


\section{Parton Bubble}

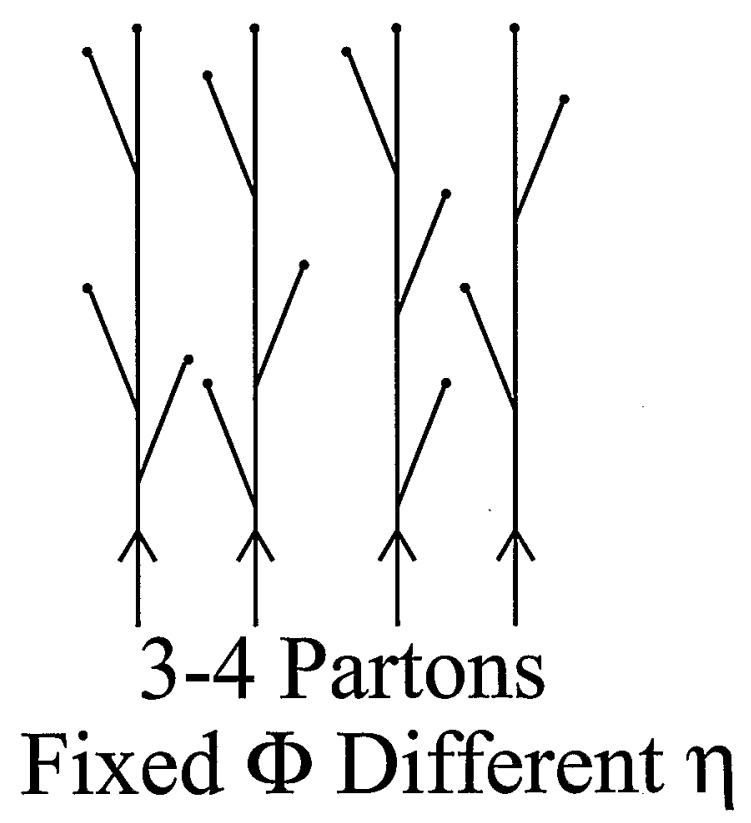

Figure 3: Each bubble contains 3-4 partons as shown. 


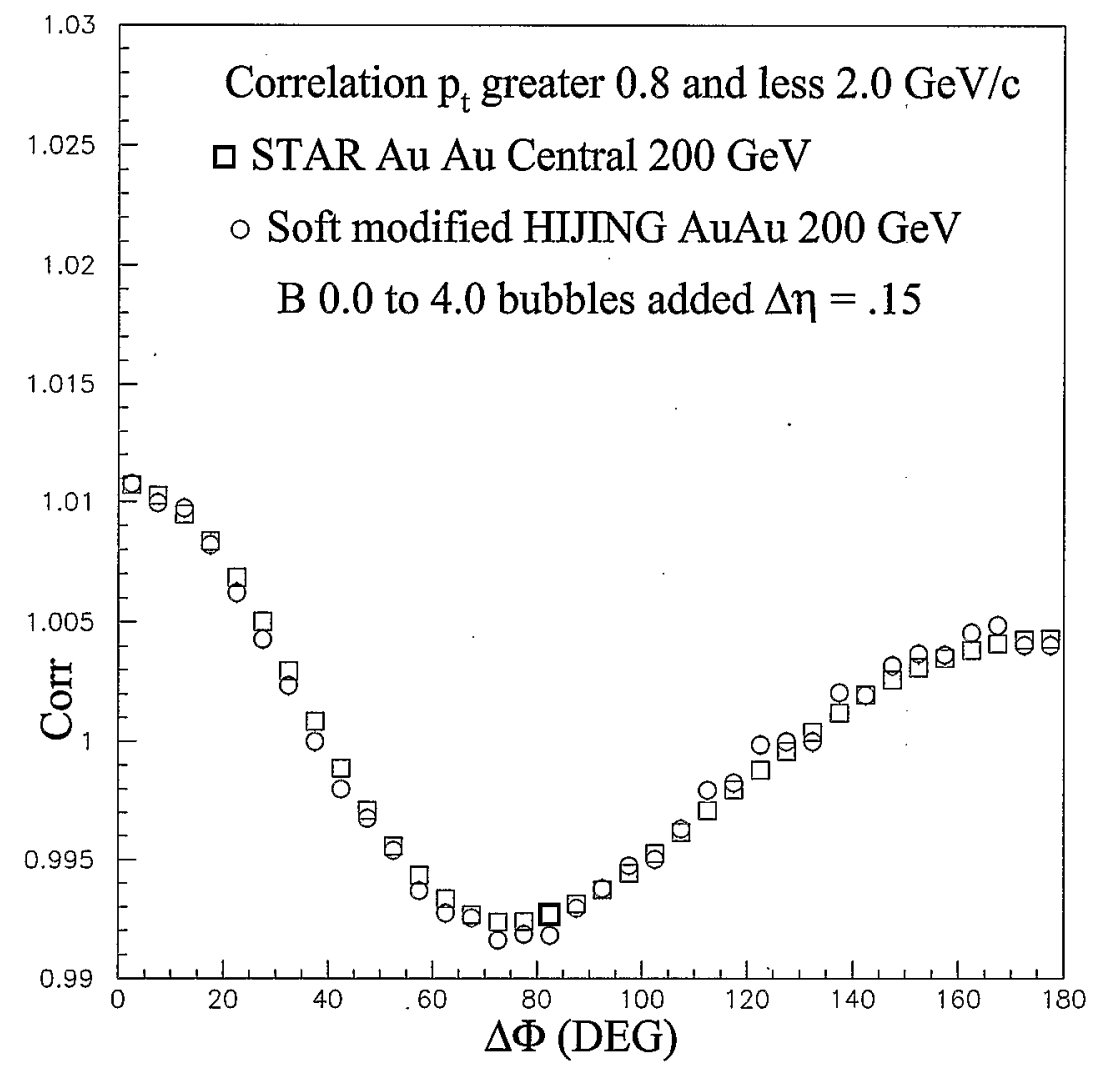

Figure 4: The $\Delta \Phi$ charged particle pair correlation for soft modified HIJING plus bubbles, which is the bubble model, compared to ref.[14] data for $\Delta \eta 0.15(0.0<\Delta \eta<0.3)$. The 0 $-10 \%$ centrality in HIJING corresponds to impact parameter (b) range 0.0 to $4.0 \mathrm{fm}$. The agreement is very good.

were generated in these simulations. The 200,000 events that were generated in the model simulations were tuned to the STAR data[14] shown in Figures 4 and 5.

Figure 4 shows the particle pair correlation for soft modified HIJING plus bubbles, which is the bubble model, compared to ref.[14] data for the $\Delta \eta$ bin $0.15(0.0<\Delta \eta<0.3)$. The $0-10 \%$ centrality region in HIJING corresponds to impact parameter (b) range 0.0 to $4.0 \mathrm{fm}$. The away side(beyond $90^{\circ}$ ) bump in Figure 4 is due to momentum conservation in the ring of bubbles plus a reaction plane generated elliptic flow v2 consistent with RHIC data(mean v2 equals 0.035$)[12]$. Figure 5 shows the model for the $\Delta \eta$ bin $0.75(0.6<\Delta \eta<0.9)$ compared to ref.[14]. The agreement between the bubble model and the RHIC data in Figures 4 and 5 is very good. 


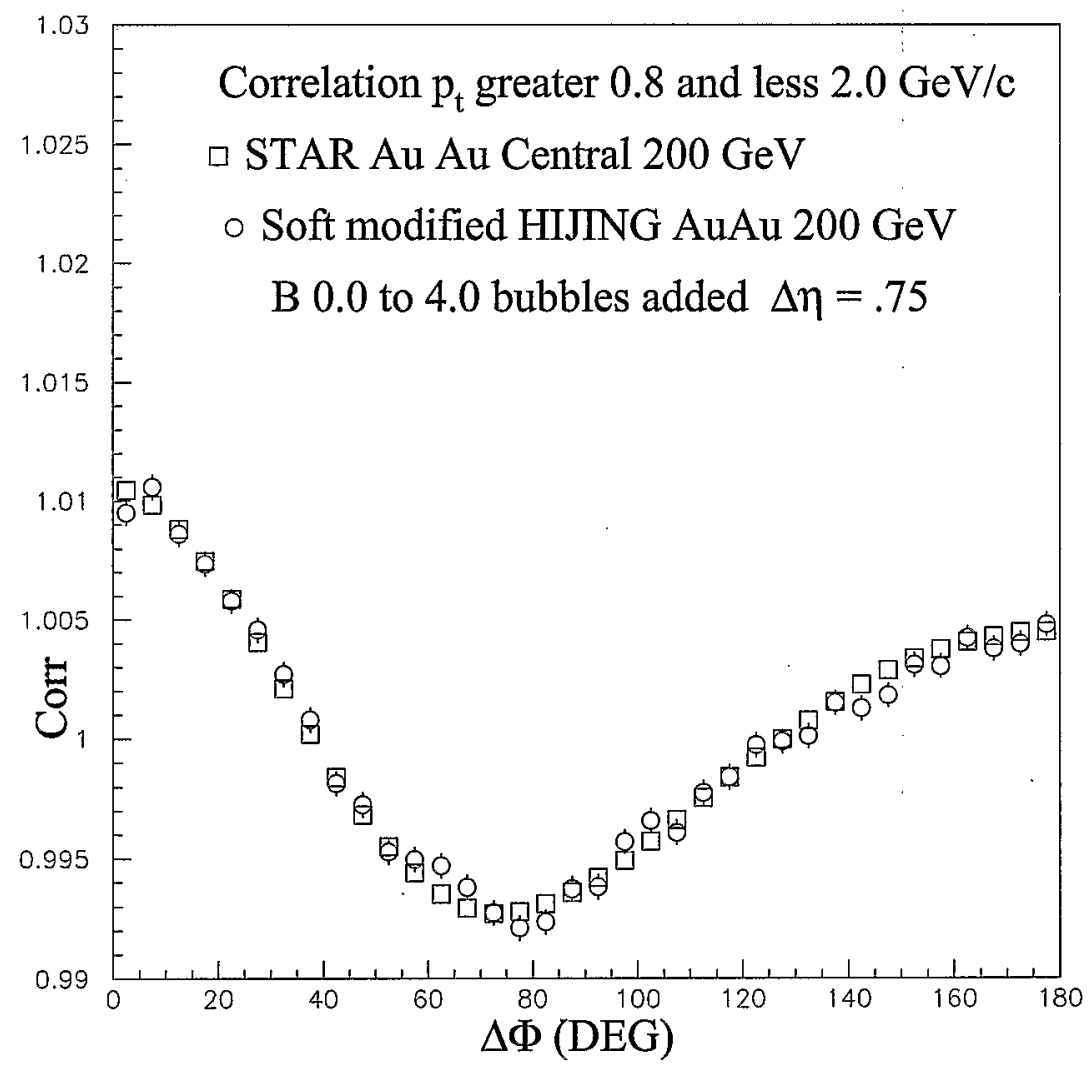

Figure 5: The $\Delta \Phi$ charged particle pair correlation for soft modified HIJING plus bubbles, which is the bubble model, compared to ref.[14] data for $\Delta \eta 0.75(0.6<\Delta \eta<0.9)$. The 0 $-10 \%$ centrality in HIJING corresponds to impact parameter (b) range 0.0 to $4.0 \mathrm{fm}$. The agreement is very good. 


\section{Parton Bubble Model Correlation Predictions}

Let us look at some correlation predictions of the bubble model we have tuned to the STAR data. First we plot the part of the correlation that comes from a single bubble considering unlike sign charged track pairs, for $|\eta|<1.0$ and $p_{t}$ from 0.8 to $4.0 \mathrm{GeV} / \mathrm{c}$. Figure 6 is a 2-dimensional (2-D) perspective plot of $\Delta \Phi$ vs. $\Delta \eta$ for the part of the unlike sign charged pair correlation signal which has contributions from particles coming from the same bubble. All other combinations of pairs are subtracted. The particles emitted from a bubble are all signal particles which contribute to correlations. The particles emitted from one bubble are uncorrelated to particles emitted from another bubble, except for momentum conservation requirements. The particles from the HIJING beam jet particles are background particles and do not contribute to the correlation. $\Phi$ is the azimuthal angle around the beam, and $\Delta \Phi$ is the difference of the azimuthal angles for the two particle pair. The pseudo-rapidity $(\eta)$ corresponds to the polar angle along the beam, and $\Delta \eta$ is the difference in $\eta$ for the two particle pair. The $\Delta \Phi$ range is $0^{\circ}$ to $180^{\circ}$ and the $\Delta \eta$ range is 0.0 to 1.5 . We see that the total width in the $\Delta \Phi$ direction is about $60^{\circ}$. The $\Delta \eta$ width is very large dropping from a high of 0.047 to 0.034 in the $\Delta \eta$ range of 0.0 to 1.5 . If this was a gaussian in $\Delta \eta$ the width would be 1.86 which would correspond to an angle of $72^{\circ}$. Our bubbles are on the surface of the fireball when they emit individual particles and resonances which decay into particles. In the center of mass (CM) of the bubble which is moving with the surface half the particles are emitted outward from the surface. These are the ones which would be observed experimentally. Particles that are emitted from the interior region of the fireball have a large probability of being absorbed by the strongly interacting medium. These particles are also softer and are mainly below our $p_{t}$ cut. The particles from the bubble that are emitted back toward the fireball would also be softer when their CM motion is subtracted from the motion in the rest frame of the bubble. Thus these particles also have a large probability of being absorbed by interaction with low momentum particles in the fireball or having their momentum reduced below our $p_{t}$ cut. We will later on in Section 6 in this paper discuss all of the particles when comparing with data that contains a large number of low energy particles, but generally we will only consider the particles above our $p_{t}$ cut which are emitted on the outgoing surface of the bubble. Figure 7 shows the like sign charged pair correlation signal coming from the same bubble. This figure is generally similar in appearance to the unlike sign charged pairs except for a symmetric dip at small $\Delta \eta$ and $\Delta \Phi$ at $\Delta \eta$ centered near zero. This dip spreads about $15^{\circ}$ in $\Delta \Phi$ and 0.3 in $\Delta \eta$ (which is approximately $15^{\circ}$ in the $\eta$ direction). In ref.[1] we had determined that the $\Delta \Phi$ width of emission of a single particle was about $30^{\circ}$, and thus the expected $\Delta \Phi$ width for a particle pair would be approximately double which is about $60^{\circ}$ in agreement with the $\Delta \Phi$ width of the unlike pairs signal and like pairs signal in Figures 6 and 7.

When we were tuning our bubble model to the RHIC data, we considered all charged pairs of tracks independent of their charge. This correlation is called the charge independent correlation (CI). The CI correlation is defined as the sum of the unlike sign charged pairs correlation and the like sign charged pairs correlation. The CI correlation is twice the correlation of all charged track pairs from a given bubble. Figure 8 shows the charge independent correlation (CI) signal for track pairs from the same bubble, all other combinations of track 


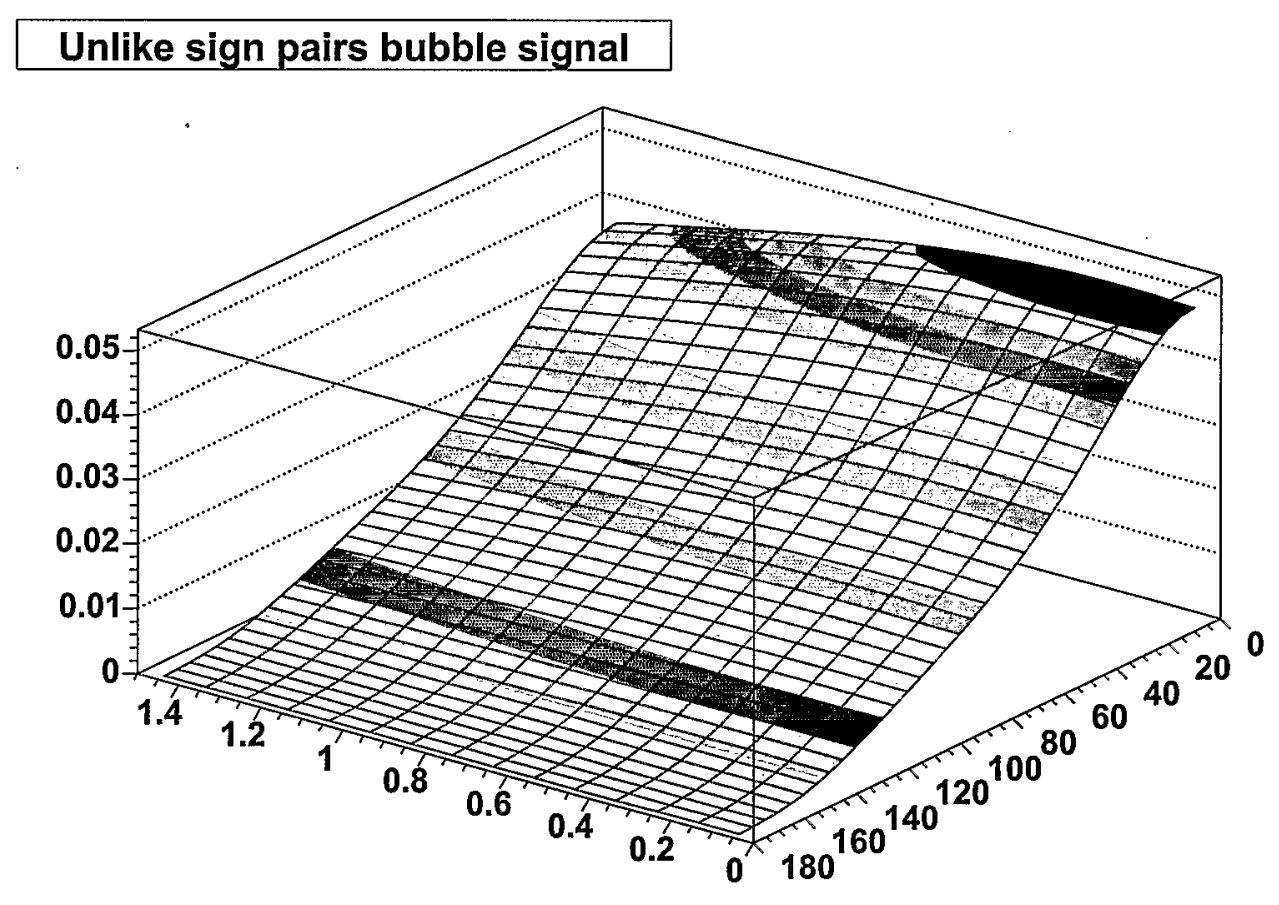

Figure 6: A 2-dimensional(2-D) perspective plot of $\Delta \Phi$ (right side scale) vs $\Delta \eta$ (left hand scale) for the part of the unlike sign charged pair correlation signal which has contributions from particles coming from the same bubble. All other combinations of pairs are subtracted. In figures 6 to 9 , because of subtraction, 0 corresponds to 0 correlation. 


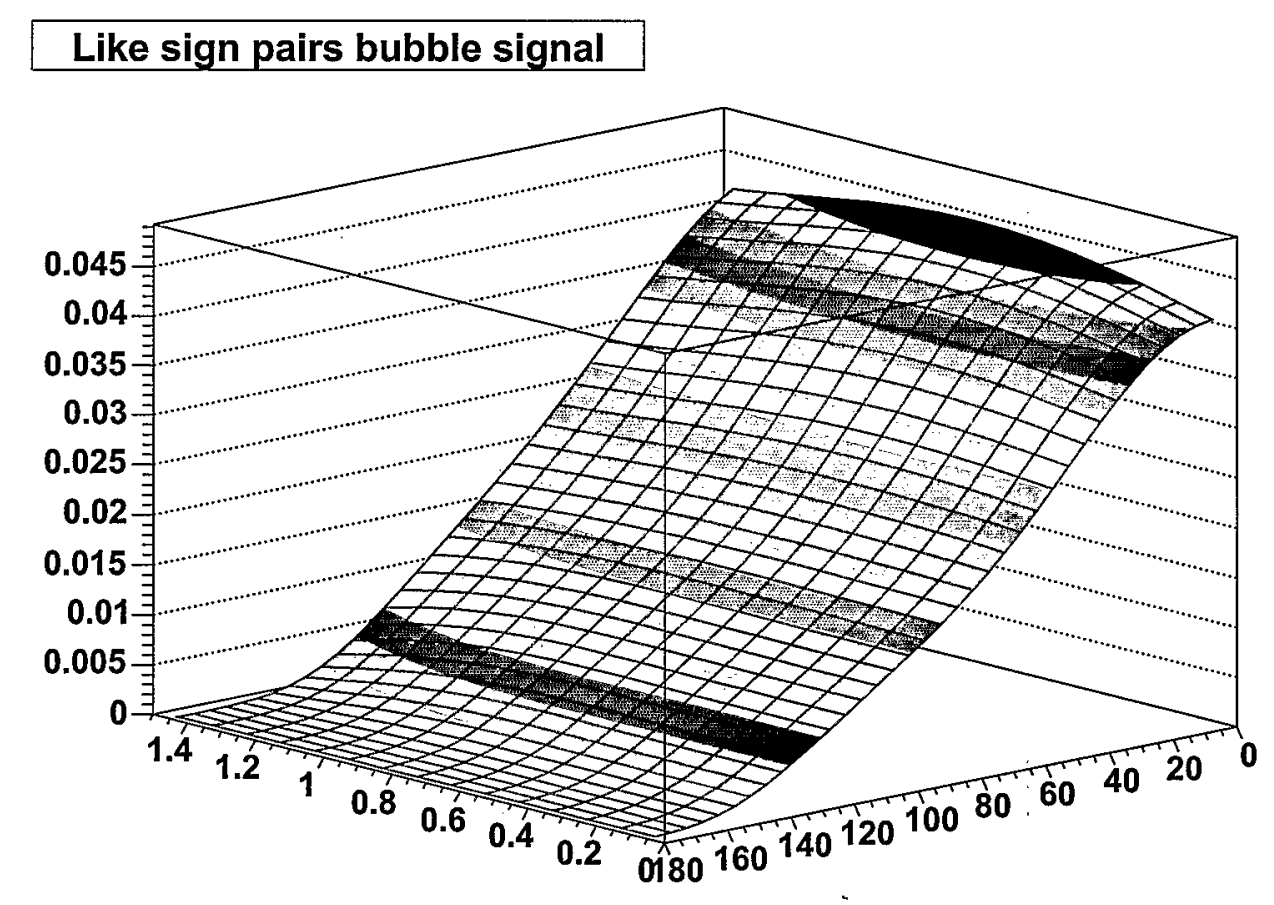

Figure 7: A 2-D perspective plot of $\Delta \Phi$ (right side scale) vs $\Delta \eta$ (left hand scale) for the part of the like sign charged pair correlation signal which has contributions from particles comimg from the same bubble. All other combinations of pairs are subtracted. 


\section{Cl Bubble signal}

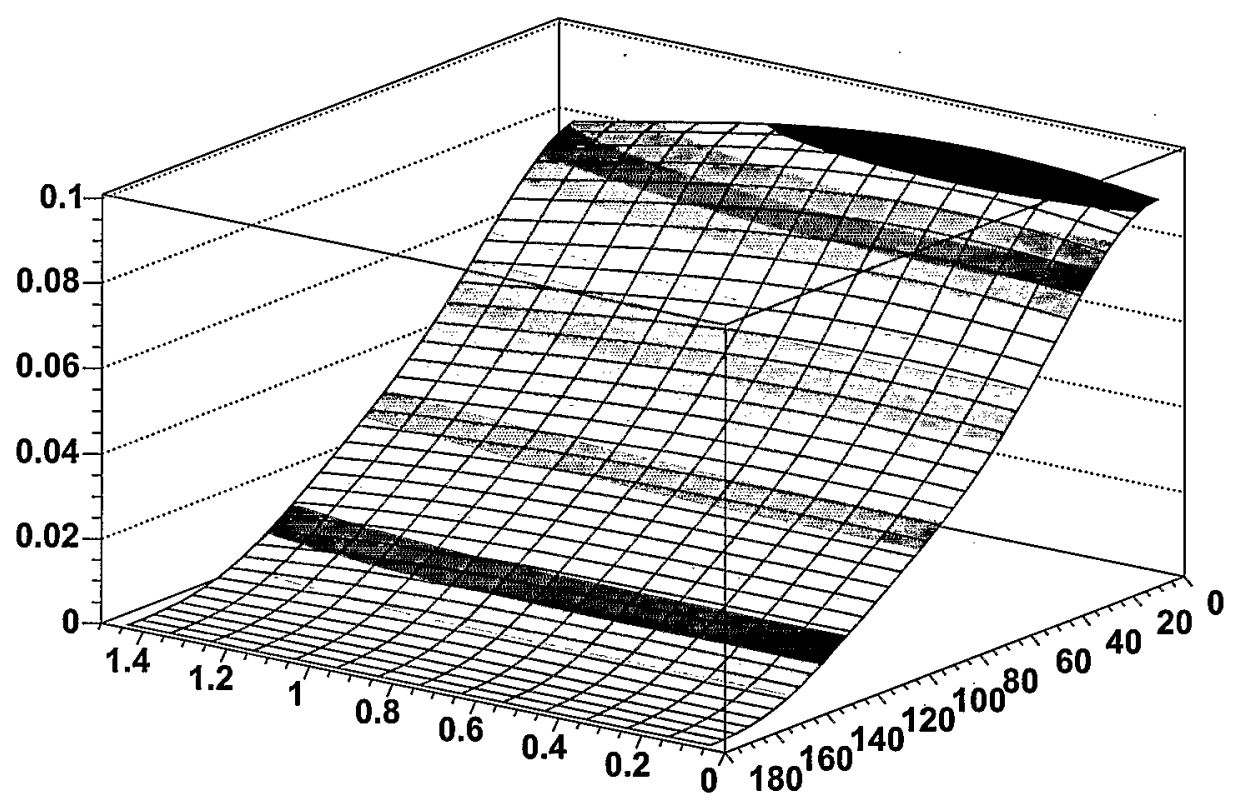

Figure 8: A 2-D perspective plot of $\Delta \Phi$ (right side scale) vs $\Delta \eta$ (left hand scale) for the charge independent correlation (CI) signal for track pairs from the same bubble. All other combinations of pairs are subtracted. This figure displays the average correlation of particles coming from individual bubbles. The emission of particles from the bubble is by pair production from partons which are almost entirely gluons.

pairs are subtracted. The HIJING background does not contribute to these correlations as previously mentioned. Again the $\Delta \Phi$ range is from $0^{\circ}$ to $180^{\circ}$ and the $\Delta \eta$ is from 0.0 to 1.5 . This figure displays the average correlation of particles coming from individual bubbles. The emission of particles from the bubble is by pair production from partons which are almost entirely gluons. The gluons produce colored pairs of quarks and anti-quarks, which recombine into color singlets that have plus, zero, or minus charge. Thus a local neutral system contains charged particles, but the total charge has to add to zero. Particle production from a local phase space cell is what creates the dip in the small $\Delta \eta$ and $\Delta \Phi$ part of the like sign charged pairs correlation in Figure 7. Each local phase space cell has on the order of one color singlet pair. Since gluons emit unlike sign charged pairs from the same phase space cell, like charged particle pairs emission from the same phase space region is suppressed.

If we subtract the like sign charged pairs signal (Figure 7) from the unlike sign charged pairs signal (Figure 6), we are left with the balancing plus and minus charge emissions. This 
correlation is called the charge dependent correlation (CD) signal. The CD correlation is defined as the subtraction of the like sign charged pairs correlation from the unlike sign charged pairs correlation, including background in each. This correlation, when observed experimentally, is within the errors the same as the CD signal which is defined as (the unlike charged pairs correlation minus the background) - (the like charged pairs correlation minus the background). This occurs since the backgrounds cancel in the subtraction.

A physical interpretation of the CD signal is that it displays a measure of the emission correlation of the opposite sign pairs of particles emitted from the same space time region. The subtraction removes those pairs of opposite sign particles which do not come from the same space time region. This CD signal correlation is shown in Figure 9. In Figure 9 the CD correlation is symmetric in the corresponding $\Delta \Phi$ and $\Delta \eta$ angular widths $\left(\sim 30^{\circ}\right)$. This is because the average pair has an opening angle between the plus and minus particle up to $60^{\circ}$ in a random distribution about some fixed $\eta$ and $\Phi$ direction for the bubble. There are some systematic errors in the fragmentation of the partons from the bubbles. To correct this we have kept the number of bubbles fixed and varied the fragmentation parameters, while keeping our agreement as good as seen in Figures 3 and 4. In Table I we give the parameters of the bubble model only considering charged particles. The neutral particles are not included in the table below.

Table I. Parameters of bubble model for charged particles.

\begin{tabular}{|c|r|r|r|}
\hline \multicolumn{4}{|c|}{ Table I } \\
\hline variable & amount & fluctuations & systematics \\
\hline bubbles & 12 & 0 & 0 \\
\hline particles & 7.3 & 2 & .7 \\
\hline soft & 104 & 12 & 0 \\
\hline$p_{t}(\mathrm{GeV} / \mathrm{c})$ & 6.0 & 0.8 & 0.3 \\
\hline energy $(\mathrm{GeV})$ & 9.0 & 4.0 & 2.0 \\
\hline
\end{tabular}

We are now in the position to make predictions about two charged particle angular correlations that should be observed at RHIC. The $\Delta \Phi$ correlations that we show in the Figure 10 through 14 are the predictions for mid rapidity central $\mathrm{Au}+\mathrm{Au}$ collisions at $\sqrt{s_{N N}}=200 \mathrm{GeV}$, where the charged particles have a $p_{t}$ range 0.8 to $4.0 \mathrm{GeV} / \mathrm{c}$ and $|\eta|$ is less than 1. Each of the five $\Delta \Phi$ correlations are done at different $\Delta \eta$ bins $0.15(0.0$ to 0.3$)$, $0.45(0.3$ to 0.6$), 0.75(0.6$ to 0.9$), 1.05(0.9$ to 1.2$)$ and $1.35(1.2$ to 1.5$)$. In each plot we show for the charged pairs the unlike, like, and the average of the unlike and like(one half of the CI).

\section{Quark Quark Recombination}

Our parton bubble has been tuned to experimental data with the results that inside the bubble there are three to four partons with differing longitudinal momenta all at the same $\dot{\Phi}$. The $p_{t}$ distribution of the partons inside the bubble is similar to pQCD but has a 


\section{Bubble signal}

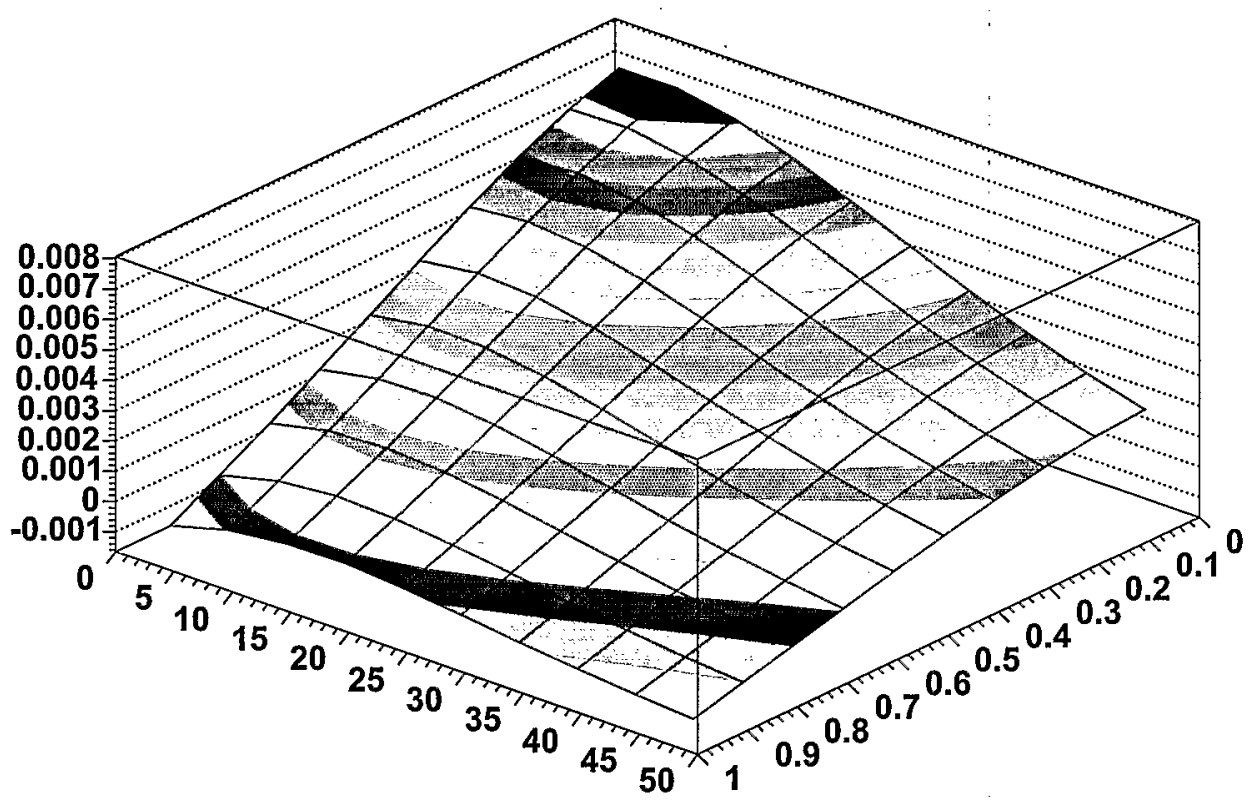

Figure 9: A 2-D perspective plot of $\Delta \Phi$ (left side scale) vs $\Delta \eta$ (right side scale) for the charge independent correlation (CD) signal for track pairs from the same bubble. All other combinations of pairs are subtracted. This figure displays a measure of the 2-D pair emission correlation of the unlike sign pairs of particles emitted from the same space-time region. The CD signal correlation is symmetric in $\Delta \Phi$ and $\Delta \eta\left(\sim 30^{\circ}\right.$ width in each). 


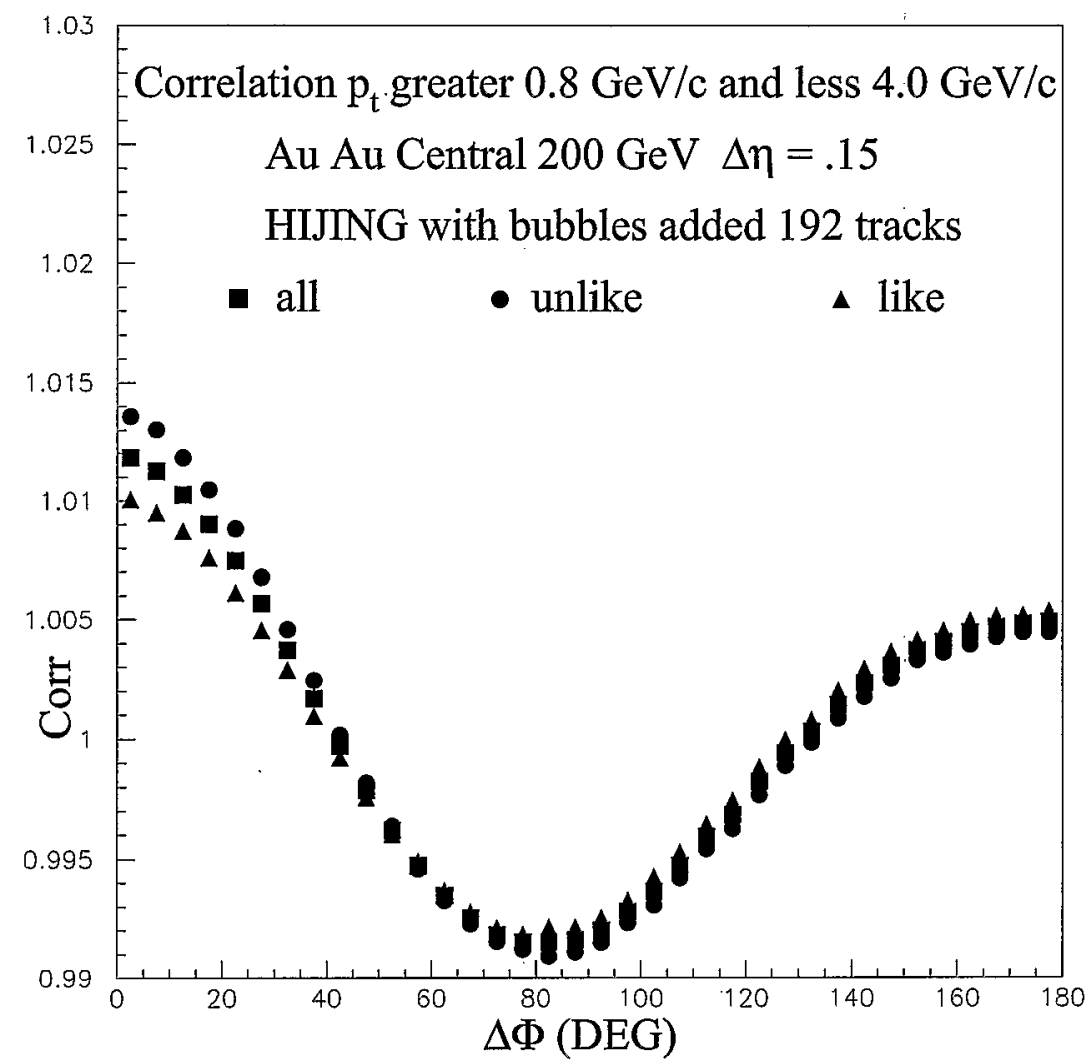

Figure 10: The bubble model prediction for the correlation as a function of $\Delta \Phi$ for the $\Delta \eta$ bin $0.15(0$ to 0.30$)$ for unlike charged pairs, like charged pairs, and all of the charged pairs. The correlation over all charged pairs is equal to average of the unlike and the like correlations and is equal to one half the CI correlation. The prominent differences are in the forward angular region which is expected for the bubble model. In this and the following figures 11-14 the label "HIJING with bubbles added" means the modified HIJING where the jets have been replaced with parton bubbles as described in the text, which is the bubble model. On this and all subsequent figures which have a correlation scale 1 corresponds to 0 correlation. 


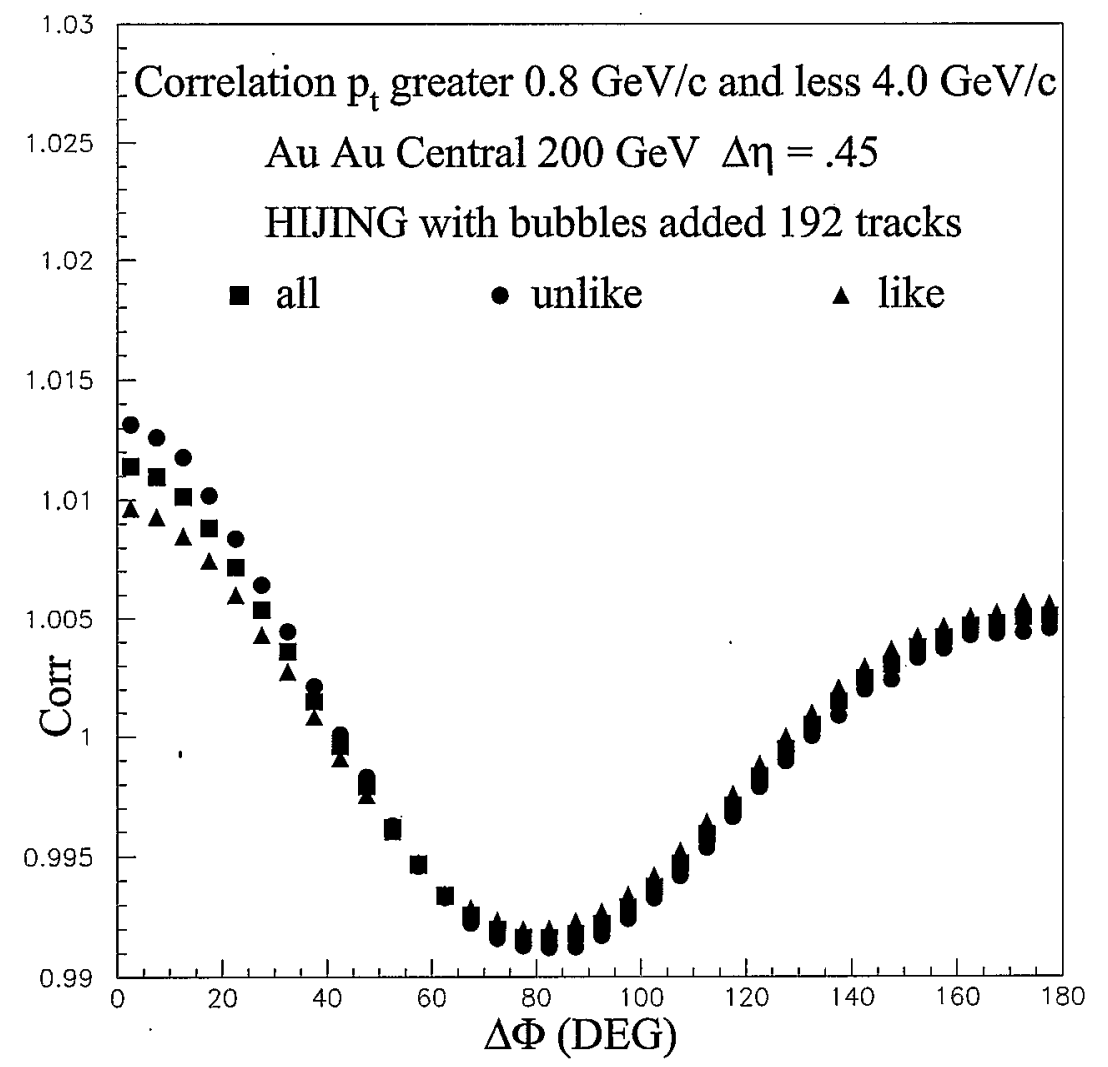

Figure 11: The bubble model prediction for the correlation as a function of $\Delta \Phi$ for the $\Delta \eta$ bin $0.45(0.30$ to 0.60$)$ for unlike charged pairs, like charged pairs, and all of the charged pairs. The correlation over all charged pairs is equal to average of the unlike and the like correlations and is equal to one half the CI correlation. The prominent differences are in the the forward angular region which is expected for the bubble model. 


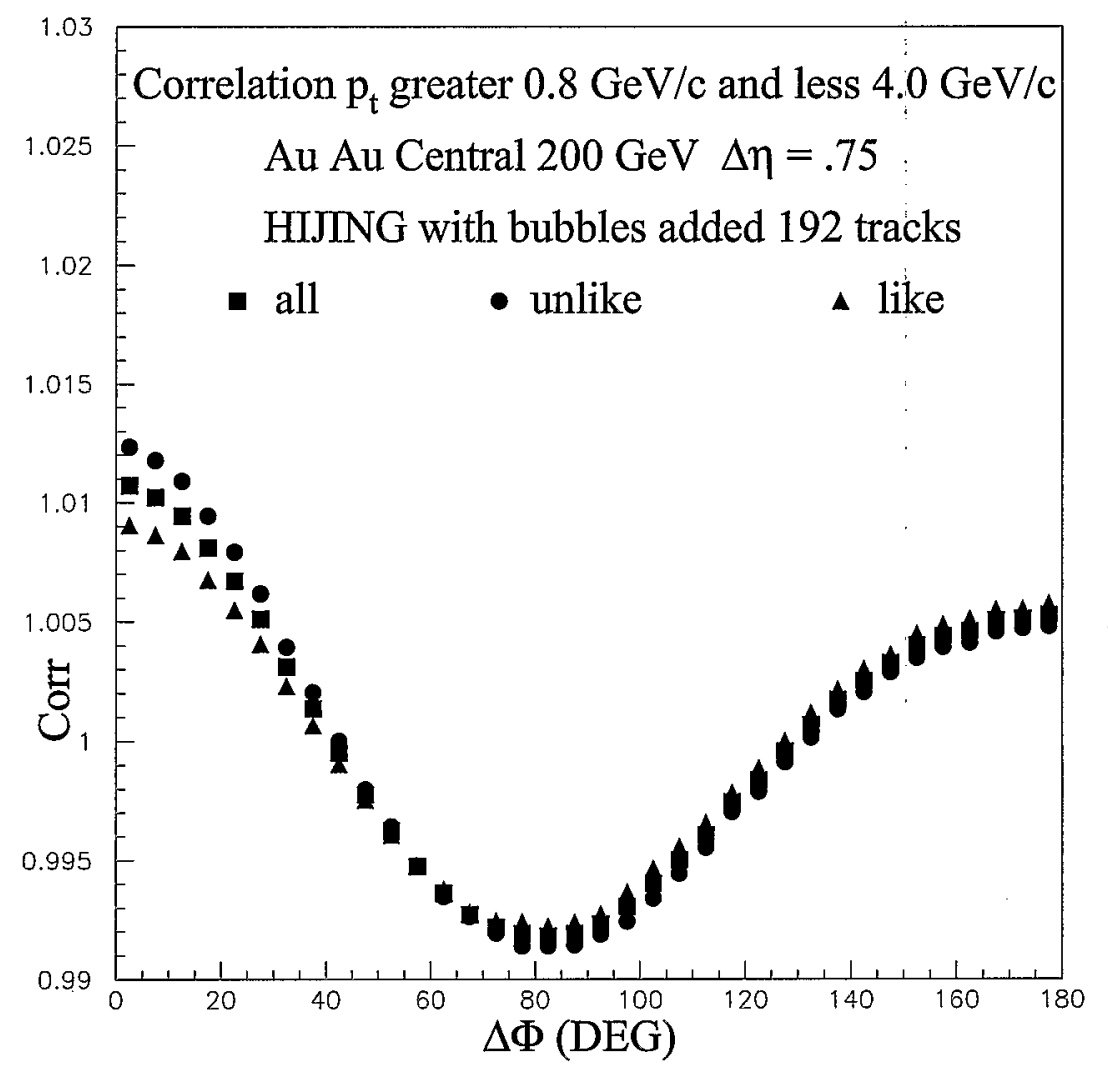

Figure 12: The bubble model prediction for the correlation as a function of $\Delta \Phi$ for the $\Delta \eta$ bin $0.75(0.60$ to 0.90$)$ for unlike charged pairs, like charged pairs, and all of the charged pairs. The correlation over all charged pairs is equal to average of the unlike and the like correlations and is equal to one half the CI correlation. The prominent differences are in the the forward angular region which is expected for the bubble model. 


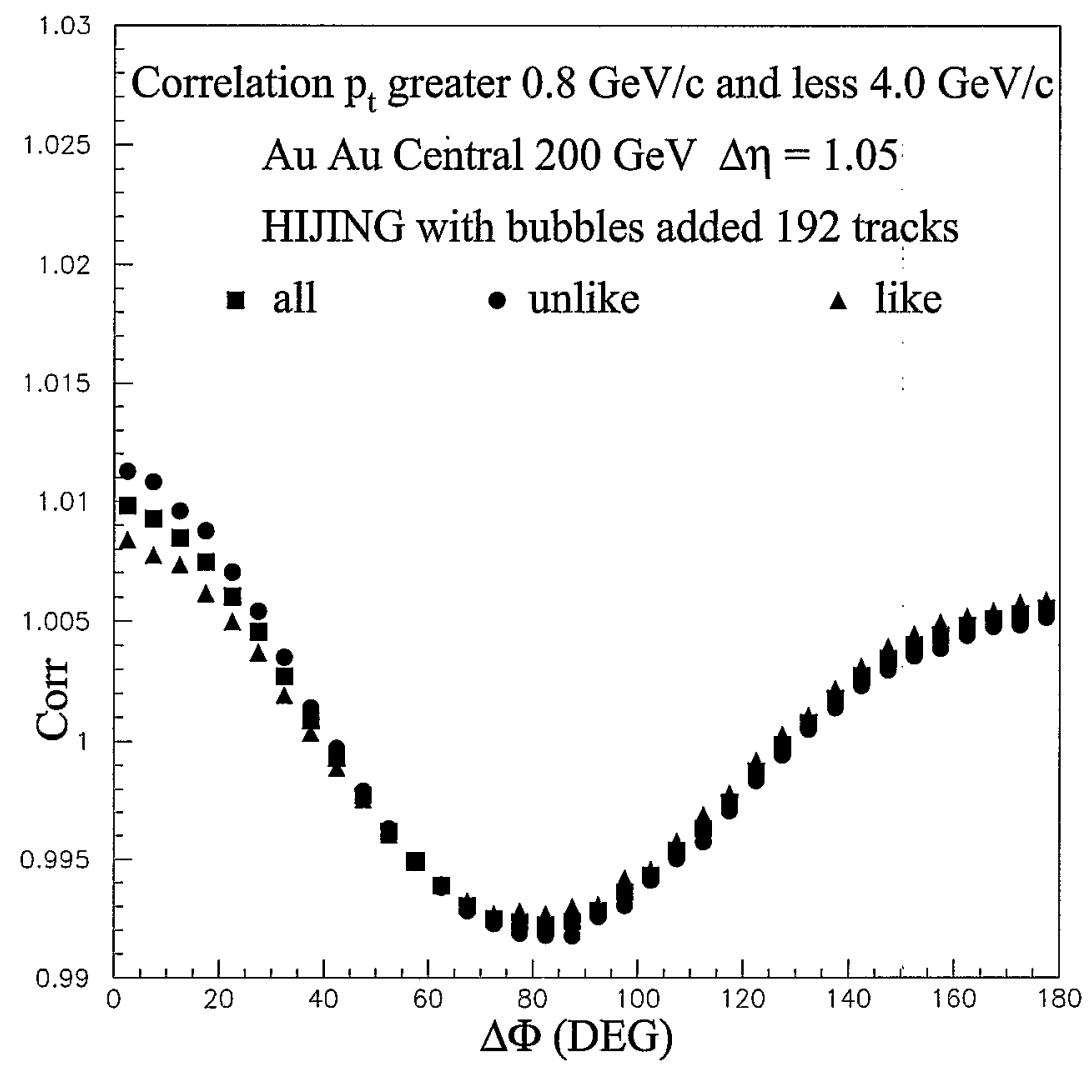

Figure 13: The bubble model prediction for the correlation as a function of $\Delta \Phi$ for the $\Delta \eta$ bin 1.05(0.90 to 1.20) for unlike charged pairs, like charged pairs, and all of the charged pairs. The correlation over all charged pairs is equal to average of the unlike and the like correlations and is equal to one half the CI correlation. The prominent differences are in the the forward angular region which is expected for the bubble model. 


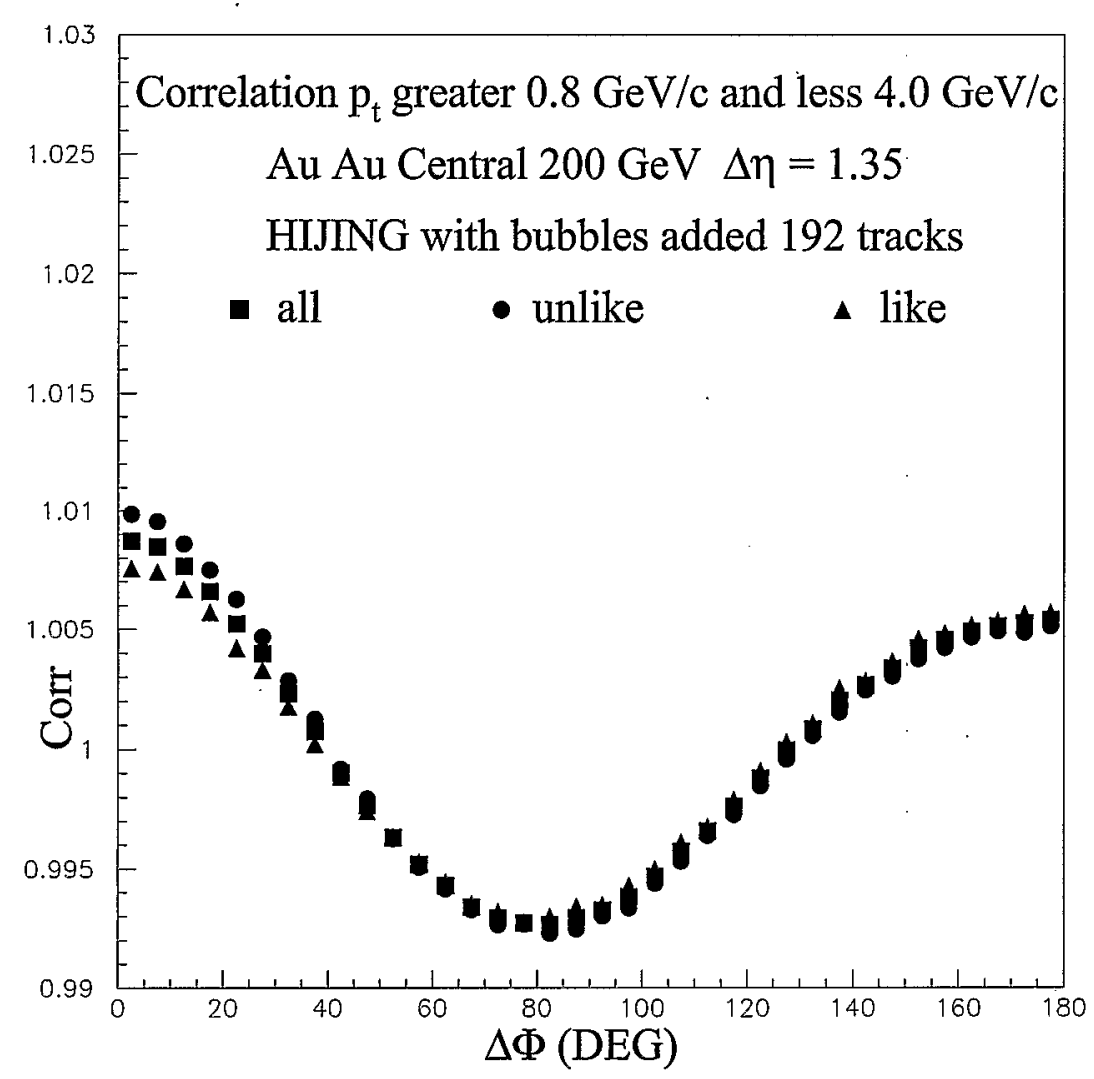

Figure 14: The bubble model prediction for the correlation as a function of $\Delta \Phi$ for the $\Delta \eta$ bin $1.35(1.20$ to 1.50$)$ for unlike charged pairs, like charged pairs, and all of the charged pairs. The correlation over all charged pairs is equal to average of the unlike and the like correlations and is equal to one half the CI correlation. The prominent differences are in the the forward angular region which is expected for the bubble model. 
suppression in the high $p_{t}$ region like the data[8]. The showering of the partons form gluons which fragment into quarks and anti-quarks which overlap with each other in space and time. This leads to and enhances the possibility that pairs of quarks from two different fragmenting partons can form a di-quark. The same process will happen for pairs of anti-quarks forming a di-anti-quark. This recombination process becomes an important possibility in our parton bubble model compared to regular jet fragmentation. Since the quarks which overlap have similar phase space, the momentum of the di-quark is approximately twice the momentum of the quarks, but has approximately the same velocity. When mesons are formed quarks pick up anti-quarks with similar phase space from fragmenting gluons to form a color singlet state. Thus the meson has approximately twice the momentum of the quark and anti-quark of which it is made. When the di-quark picks up a quark and forms a color singlet it will have approximately three times the momentum of one of the three quarks it is made from. Thus we expect a $p_{t}$ spectrum scaling when we compare mesons to baryons. Figure 15 shows the ratio protons plus anti-protons to charged particle as a function of $p_{t}$ for particles in our simulated central $\mathrm{Au}+\mathrm{Au}$ collisions. In Figure 15 we also plot the ratio from central $\mathrm{Au}+$ Au RHIC data[15]. These experimental results agree well (considering the errors) with the bubble model predictions for all charged particles. The background particles from HIJING have the same ratios observed in $\mathrm{p} \mathrm{p}$ collisions, while particles coming from our bubbles have a much larger ratio.

Recombination is an important aspect of our bubble model and leads to, for example a prediction for the proton and anti-proton tagged spectrum. We form $\Delta \Phi$ correlations for our usual five $\Delta \eta$ bins using a proton or anti-proton tagged as our reference. The $\Delta \Phi$ is measured from the tagged proton or anti-proton to another charged particle(this particle can also be a proton or anti-proton). The $\Delta \Phi$ is folded to lie between $0^{\circ}$ to $180^{\circ}$. The delta eta is in done the same way folded about $\Delta \eta$ equal zero. Figure 16 shows the $\Delta \Phi$ correlation for our five standard $\Delta \eta$ bins $(0.15(0.0$ to 0.3$), 0.45$ (0.3 to 0.6$), 0.75$ (0.6 to 0.9$), 1.05$ (0.9 to 1.2$)$ and $1.35(1.2$ to 1.5$))$. By comparison we find that we predict that this correlation is only slightly stronger than our CI correlation for all charged particles. Of course correlations of other two particle combinations can be predicted by the model.

\section{Parton Bubble Model compared to standard HIJING}

In this section we make a comparison with standard HIJING[11] with jet quenching turned off. With quenching off HIJING gives an inclusive $p_{t}$ spectrum of charged particles above $3.0 \mathrm{GeV} / \mathrm{c}$ which is consistent with binary scaling of ref.[8]. In ref[1] we found that expected QCD jets from HIJING at $\sqrt{s_{N N}}=130 \mathrm{GeV}$ for $0-10 \%$ centrality $\mathrm{Au}+\mathrm{Au}$ led to a scale factor which was about half that from the bubble model. This was confirmed when we doubled the number of jets in HIJING. However the width of jets in $\Delta \eta$ is much narrower for HIJING (or QCD jets) than for bubbles. In ref.[14] we showed a comparison of STAR $\sqrt{s_{N N}}=200 \mathrm{GeV}$ correlation data for $0-10 \%$ centrality $\mathrm{Au}+\mathrm{Au}$ with HIJING that also had twice the jets added. From this reference we compared the $\Delta \Phi$ correlation for two $\Delta \eta$ bins $(0.15(0.0$ to 0.3$)$ and $0.7(0.6$ to 0.9$))$. Again the width in $\Delta \eta$ of the jets was much less compared to the data that we adjusted our bubble model to. 


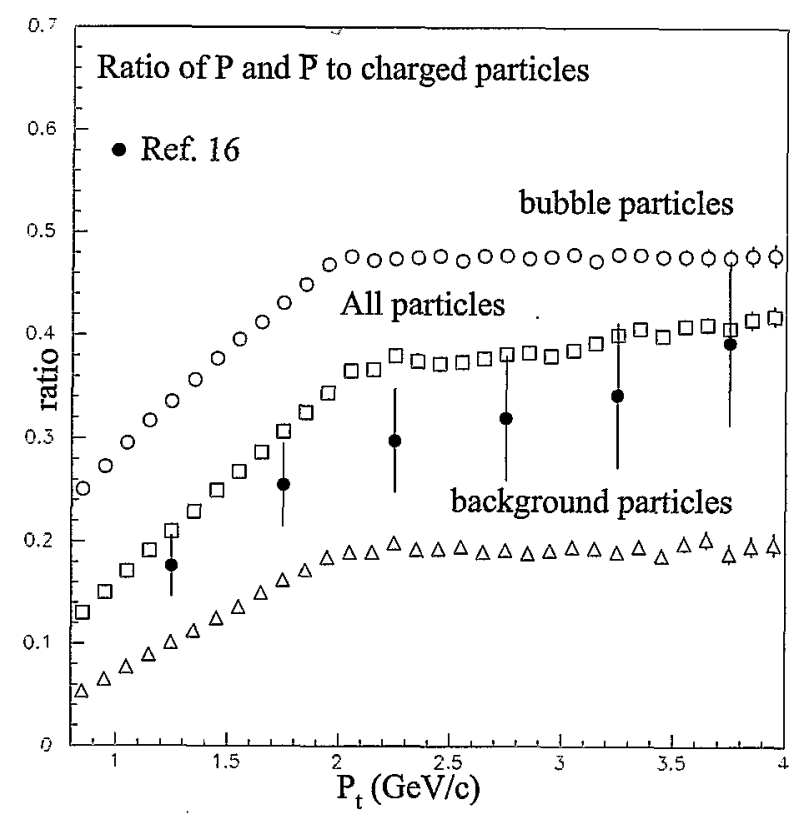

Figure 15: Shows the ratio of protons plus anti-protons to charged particles as a function of $p_{t}$ for particles in our simulated central $\mathrm{Au}+\mathrm{Au}$ collisions. We also plot the ratio from central $\mathrm{Au}+\mathrm{Au}$ RHIC data[15]. These experimental results agree well (considering the errors) with the bubble model predictions for all charged particles. The plotted ratio for the background particles in HIJING is similar to $\mathrm{p} p$ collisions. 


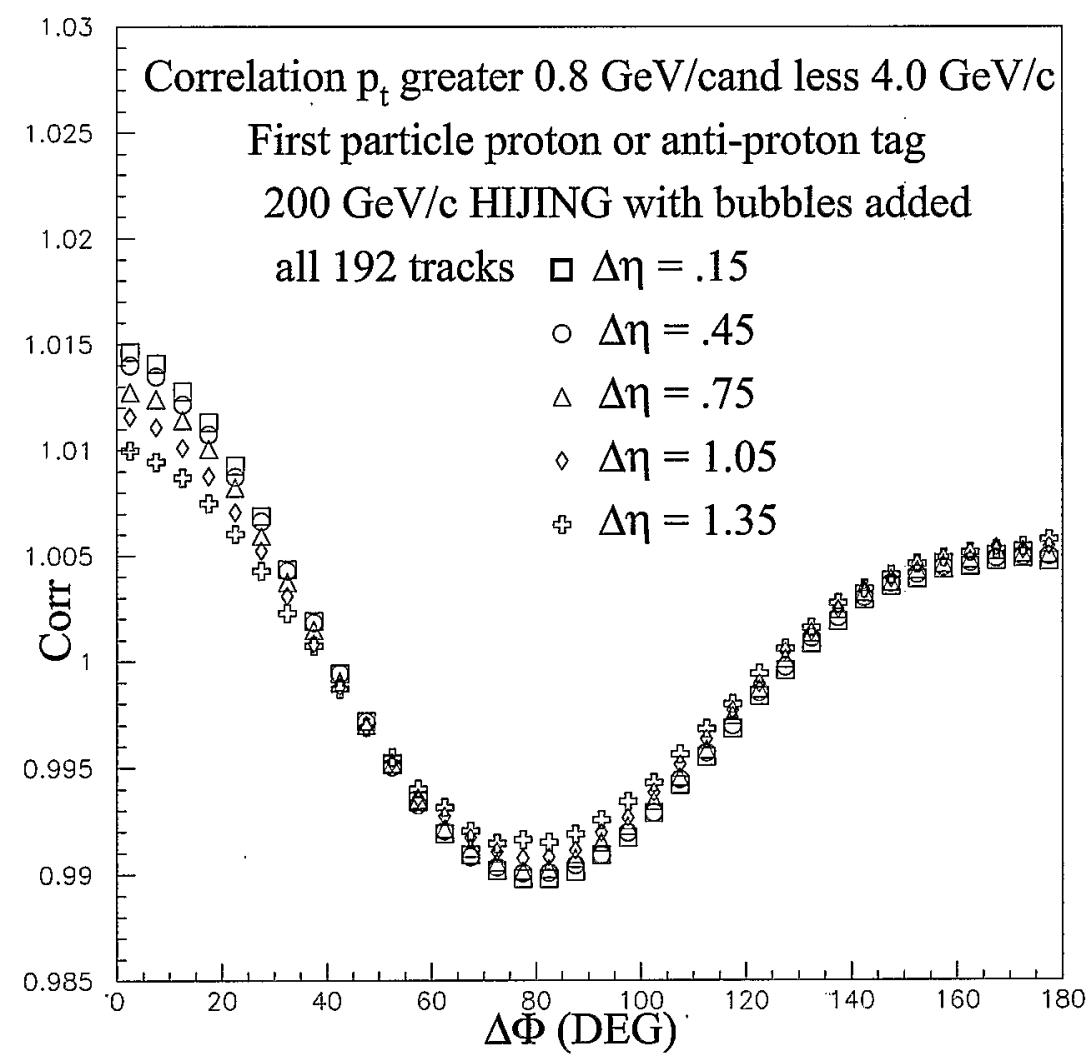

Figure 16: Predicted correlation for $\Delta \Phi$ in the $\Delta \eta=0.15$ bin, $\Delta \eta=0.45$ bin, $\Delta \eta=0.75$ bin, $\Delta \eta=1.05$ bin, and $\Delta \eta=1.35$ bin, when the first particle is tagged as a proton or anti-proton. 
In this section we have chosen to make a comparison of our bubble model with the standard HIJING (with no jet quenching) for all of our $\Delta \eta$ bins and for the two particle CI $\Delta \Phi$ correlations. Figures 17 through 21 show the two particle CI $\Delta \Phi$ correlation for our five standard $\Delta \eta$ bins $(0.15(0.0$ to 0.3$), 0.45(0.3$ to 0.6$), 0.75(0.6$ to 0.9$), 1.05(0.9$ to 1.2$)$ and 1.35(1.2 to 1.5)). The HIJING results differ greatly from the bubble model predictions.

Let us compare HIJING jet properties (Table II) to our bubble model. We have only 12 bubbles compared to the 26 jets of HIJING which is a mere factor of 2 . Each jet on the average has 3 charged particles compared to 7 from a bubble, another of factor 2 . Thus the number of charged particles from correlated objects is about the same 78 compared to 88 . However the bubble model (modified HIJING with bubbles replacing the jets) in Figures 17 to 21 has a much larger correlation, near $\Delta \Phi$ and $\Delta \eta$ equal 0 , and has a much larger $\Delta \eta$ width than jets. The above facts are understood when we consider the total energy in charged particles from jets is $57 \mathrm{GeV}$ and is $116 \mathrm{GeV}$ for bubbles (more than twice as much). Since the charged particles that fragment from jets are at close to the same $\eta$ (modest spread in $\Delta \eta$ ) the energy per jet is approximately the same as the sum $p_{t}$ per jet.

Table II. Parameters of HIJING jets for charged particles.

\begin{tabular}{|c|r|}
\hline \multicolumn{2}{|c|}{ Table II } \\
\hline variable & amount \\
\hline jets & 26 \\
\hline particles & 3 \\
\hline soft & 114 \\
\hline$p_{t}(\mathrm{GeV} / \mathrm{c})$ & 2.2 \\
\hline energy $(\mathrm{GeV})$ & 2.2 \\
\hline
\end{tabular}

\section{Soft Particles from Bubbles on the Surface}

We have chosen our $p_{t}$ range in order to resolve the individual bubbles on the surface of the expanding fireball of the $\mathrm{Au}+\mathrm{Au}$ collision. We have assumed that particles from a lower $p_{t}$ range mainly come from inside the fireball since the HBT radii of soft pions is around $6 \mathrm{fm}$. However there are charged particles that would come from the bubble which are below the $0.8 \mathrm{GeV} / \mathrm{c} p_{t}$ cut which was imposed. Many of these particles could be mixed in with the soft particles from the fireball, and could easily undergo re-scattering with the soft particles, and lose their correlation with each other and with particles which are above $0.8 \mathrm{GeV} / \mathrm{c}$. On the average each bubble creates 45 charged particles, where only 7 are above $0.8 \mathrm{GeV} / \mathrm{c}$. The average sum $p_{t}$ of the bubble is $11 \mathrm{GeV} / \mathrm{c}$ with $6 \mathrm{GeV} / \mathrm{c}$ or $58 \%$ coming from the 7 particles above $0.8 \mathrm{GeV} / \mathrm{c}$. The total rest frame energy from the 45 charged particles is 27 $\mathrm{GeV}$ which is three times the $9 \mathrm{GeV}$ that goes into the 7 particles above $0.8 \mathrm{GeV} / \mathrm{c}$. Thus above our $p_{t}$ cut we detect $58 \%$ of the momentum and $33 \%$ of the energy of the bubble.

Ref.[16] has fitted $\mathrm{Au}+\mathrm{Au}$ CI $\Delta \Phi$ vs $\Delta \eta$ correlations for a $p_{t}$ range 0.15 to $2.0 \mathrm{GeV} / \mathrm{c}$. It is possible to compare these results with our bubble model under the assumption that none 


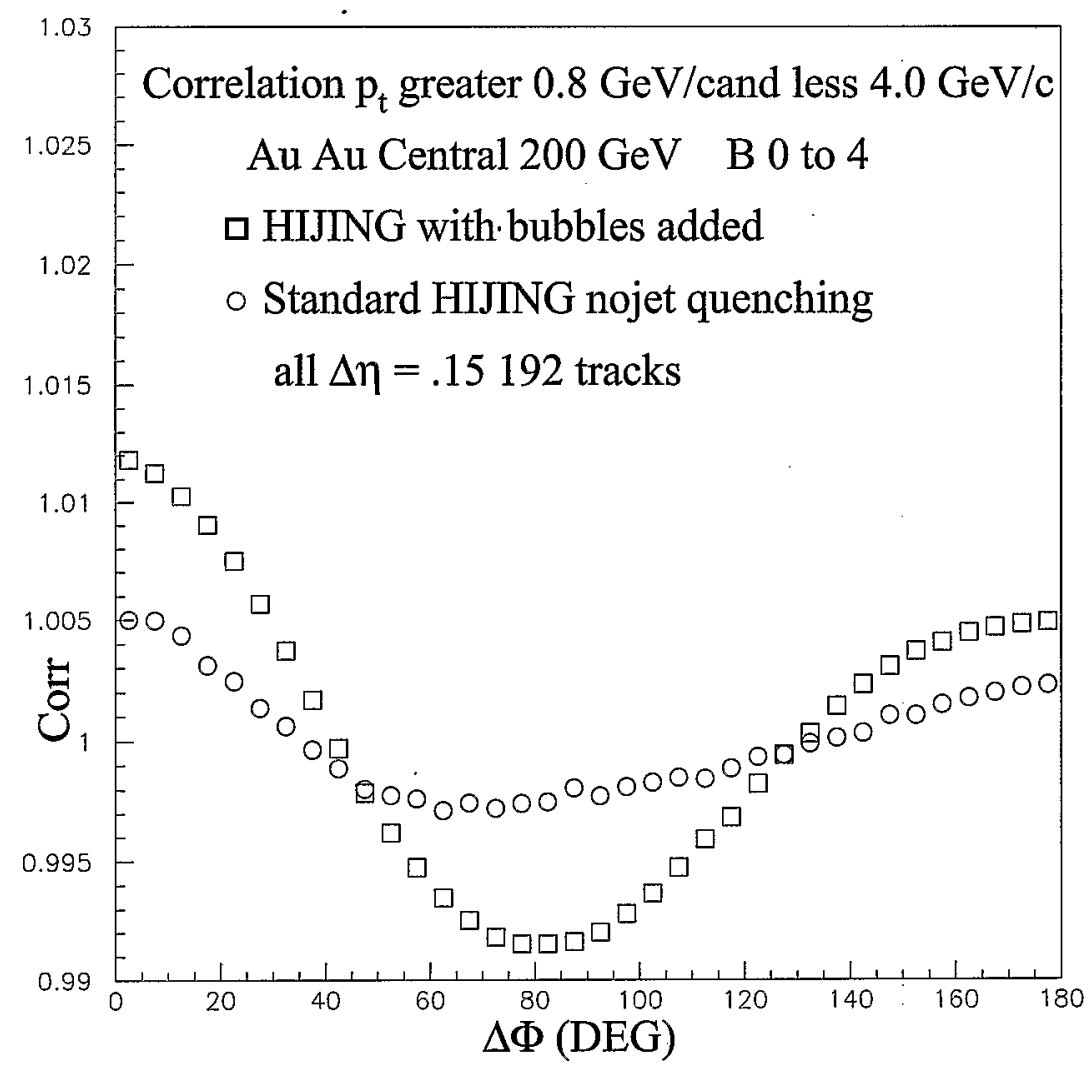

Figure 17: shows the bubble model two particle CI correlation compared to standard HIJING (without quenching) $\mathrm{CI}$, for the $\Delta \eta=0.15$ bin. 


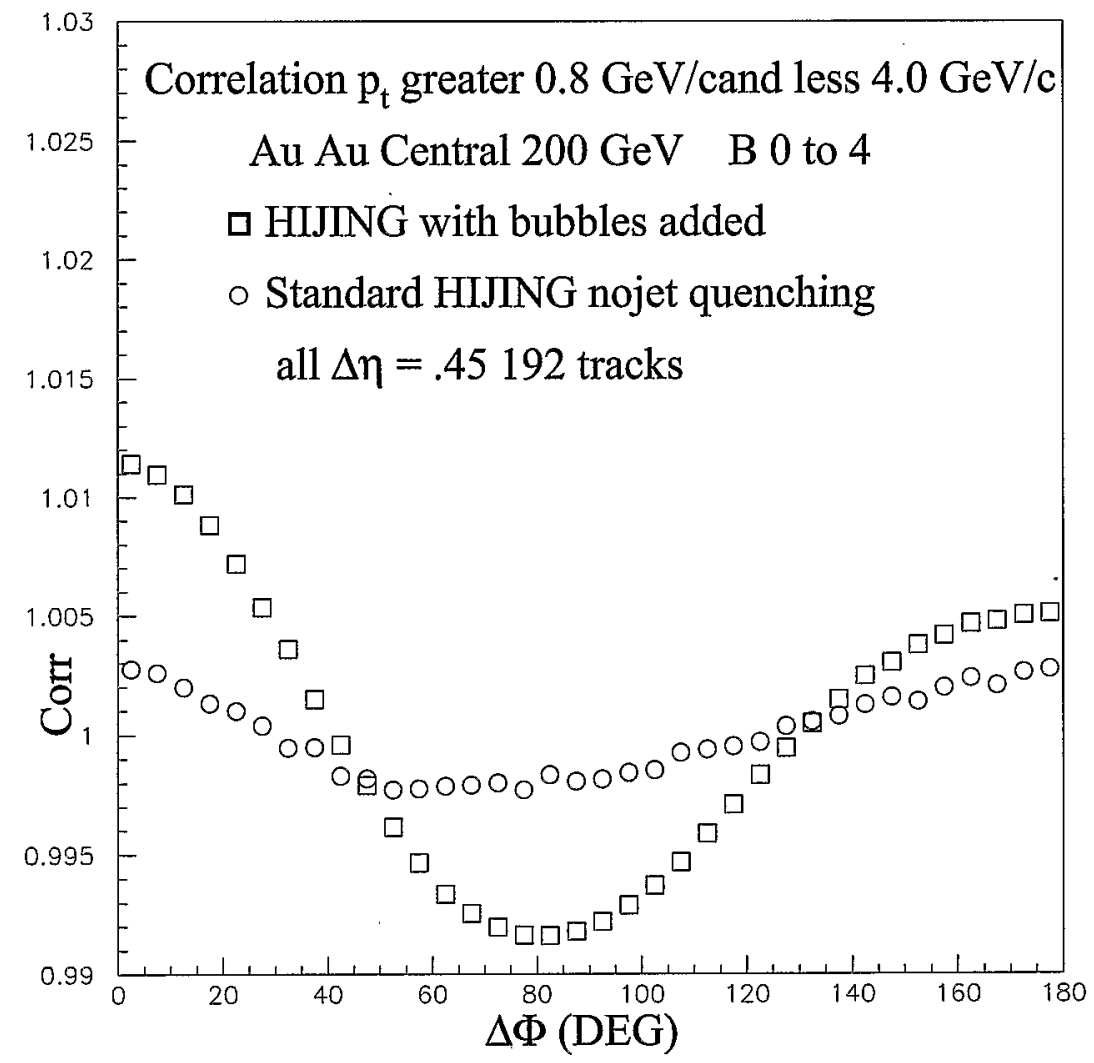

Figure 18: shows the bubble model two particle CI correlation compared to standard HIJING (without quenching) CI, for the $\Delta \eta=0.45$ bin. 


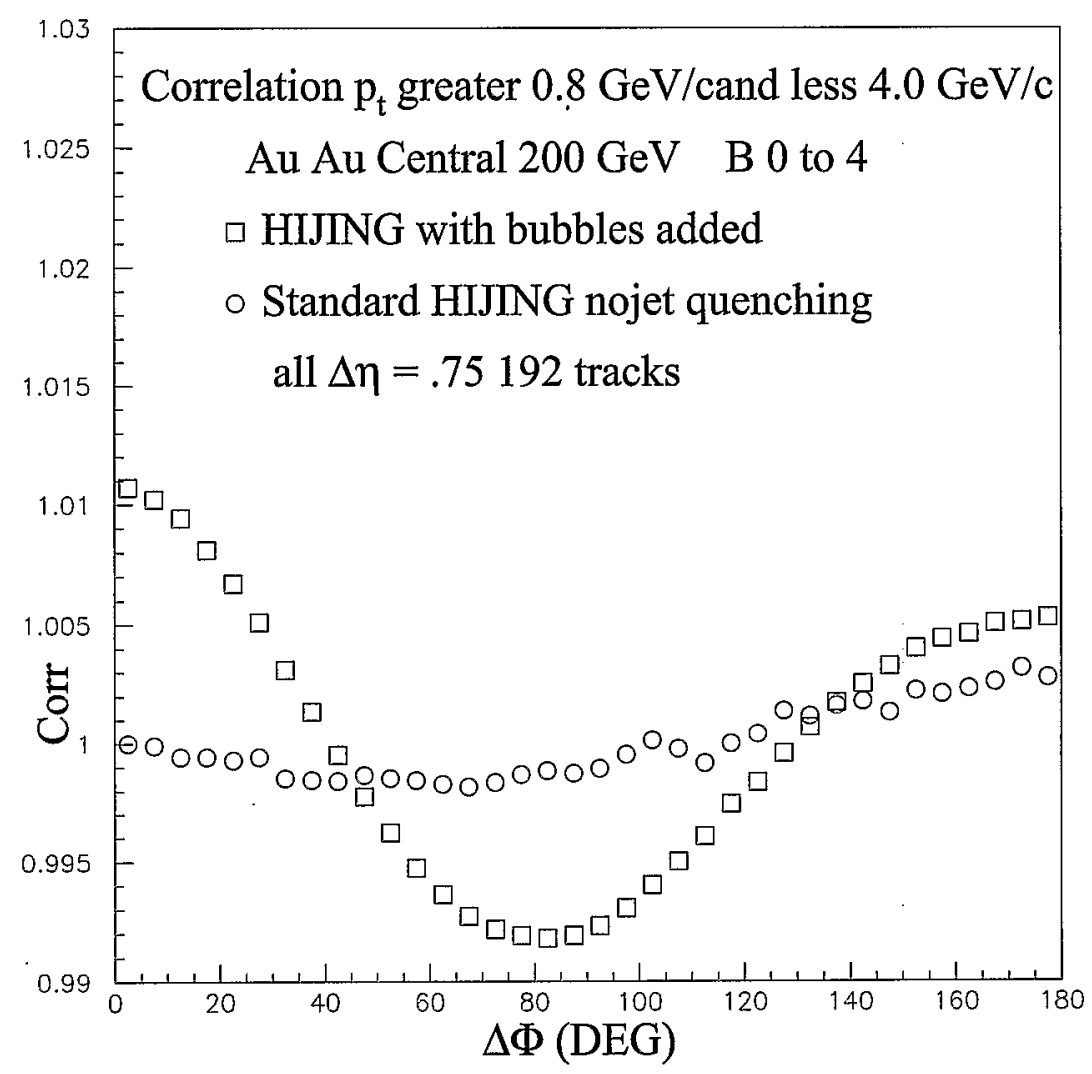

Figure 19: shows the bubble model two particle CI correlation compared to standard HIJING (without quenching) CI, for the $\Delta \eta=0.75$ bin. 


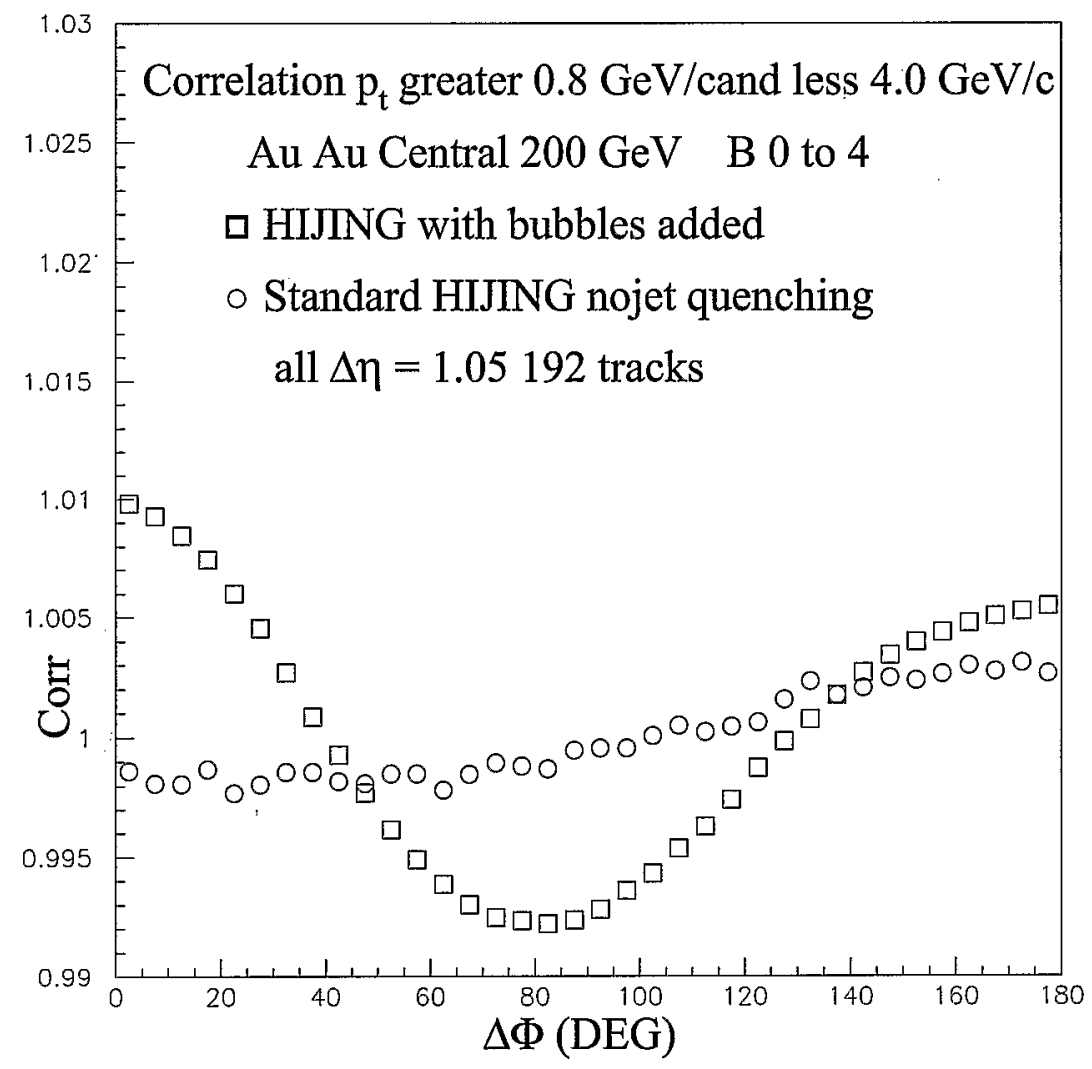

Figure 20: shows the bubble model two particle CI correlation compared to standard HIJING (without quenching) $\mathrm{CI}$, for the $\Delta \eta=1.05$ bin. 


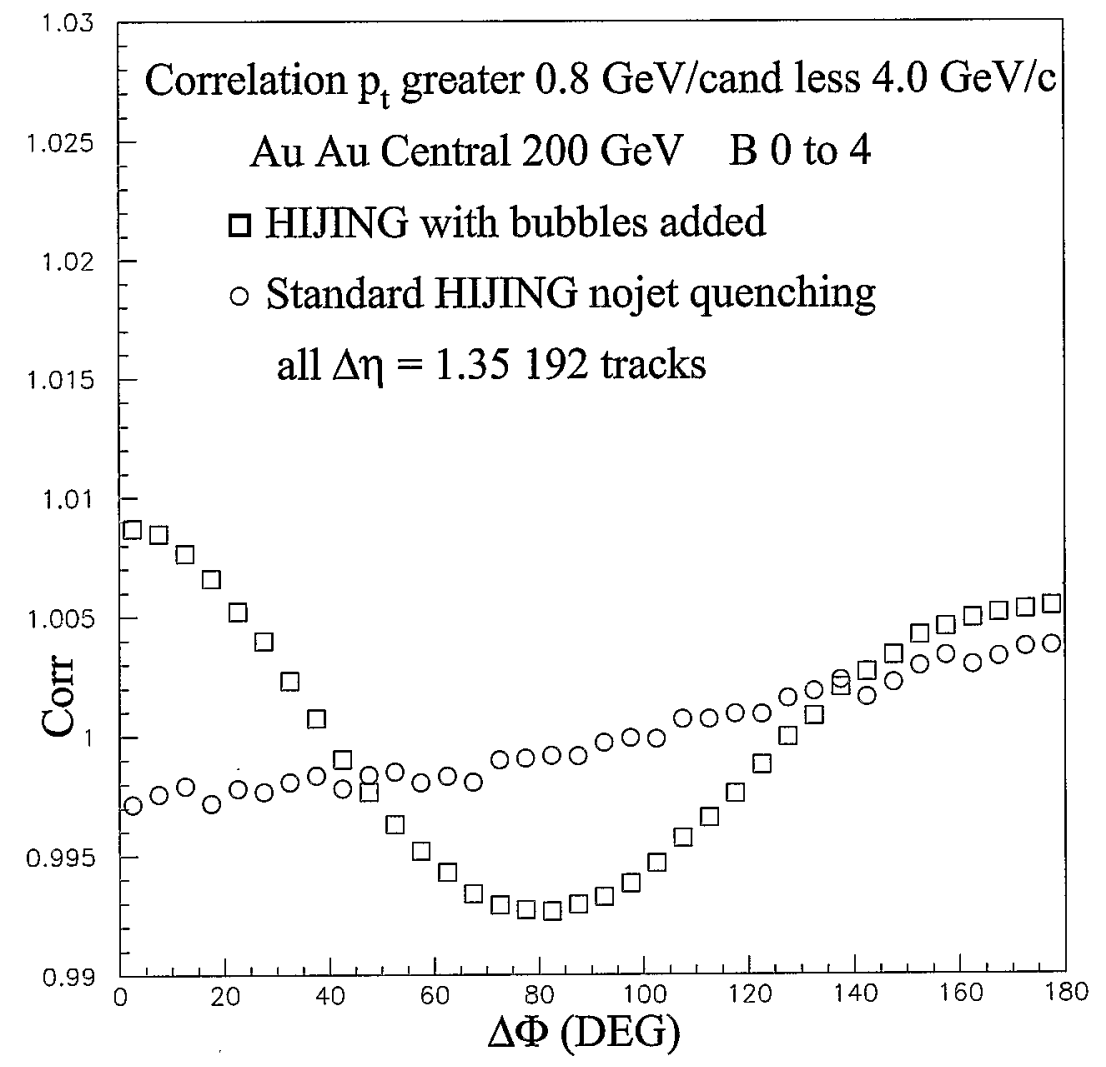

Figure 21: shows the bubble model two particle CI correlation compared to standard HIJING (without quenching) CI, for the $\Delta \eta=1.35$ bin. 


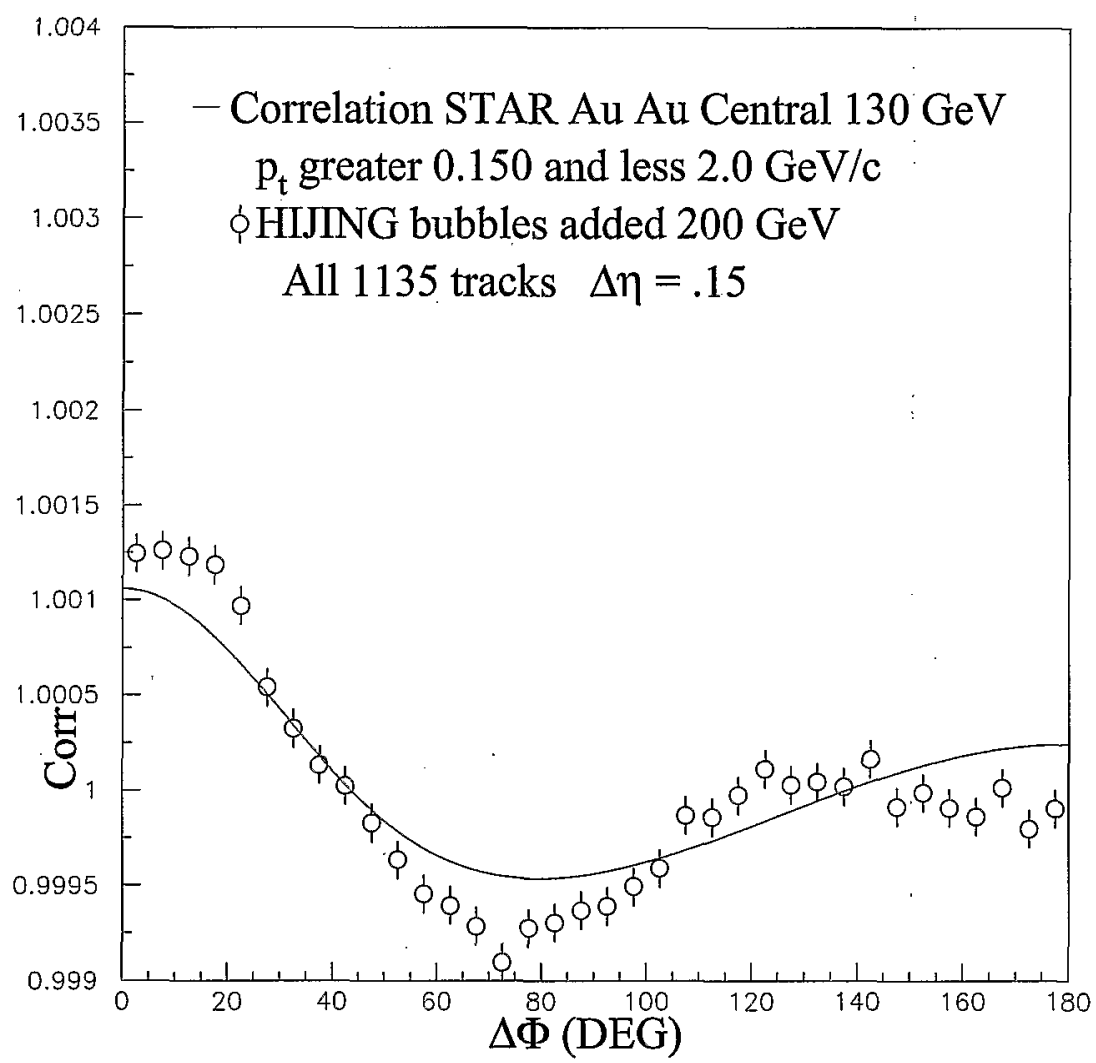

Figure 22: Comparison of the CI correlation in the $\Delta \eta=0.15$ bin of the bubble model prediction for $\sqrt{s_{N N}}=200 \mathrm{GeV}$ with experimental results at $\sqrt{s_{N N}}=130 \mathrm{GeV}$. See section VI of the text for approximations made and discussion.

of our soft charged particles interact in the final state. We believe that this would be highly unlikely and such a comparison would be an upper limit to the correlation from the lower $p_{t}$ particles. If the observed correlation is larger than our upper limit then there must be more sources of correlated particles. In this comparison we are not considering the possibility that bubbles which exist inside the fireball may also contribute some angular correlations. We are only considering surface bubbles which emit charged particles after freezeout, and that do not interact in the final state. The 45 charged particles per bubble times 12 bubbles approximately equals 540 particles that are added to the soft particles from HIJING beam jets. If we use the $p_{t}$ range of ref.[16], we obtain 535 charged particles from bubbles resulting in on the average 1135 charged particles per event when the soft particles of the beam jets are added. Figures 22 through 26 show the two particle CI $\Delta \Phi$ correlation for our five standard $\Delta \eta$ bins $(0.15$ (0.0 to 0.3$), 0.45$ (0.3 to 0.6$), 0.75(0.6$ to 0.9$), 1.05(0.9$ to 1.2$)$ and $1.35(1.2$ to 1.5)). The curves are from ref.[16] where the fits were done on mid rapidity $\mathrm{Au}+\mathrm{Au}$ central events at $\sqrt{s_{N N}}=130 \mathrm{GeV}$. Here we have compared the results of our bubble model with the experimental $\sqrt{s_{N N}}=130 \mathrm{GeV}$ correlation which does not change a lot from $\sqrt{s_{N N}}=$ $200 \mathrm{GeV}$ to $130 \mathrm{GeV}$. 


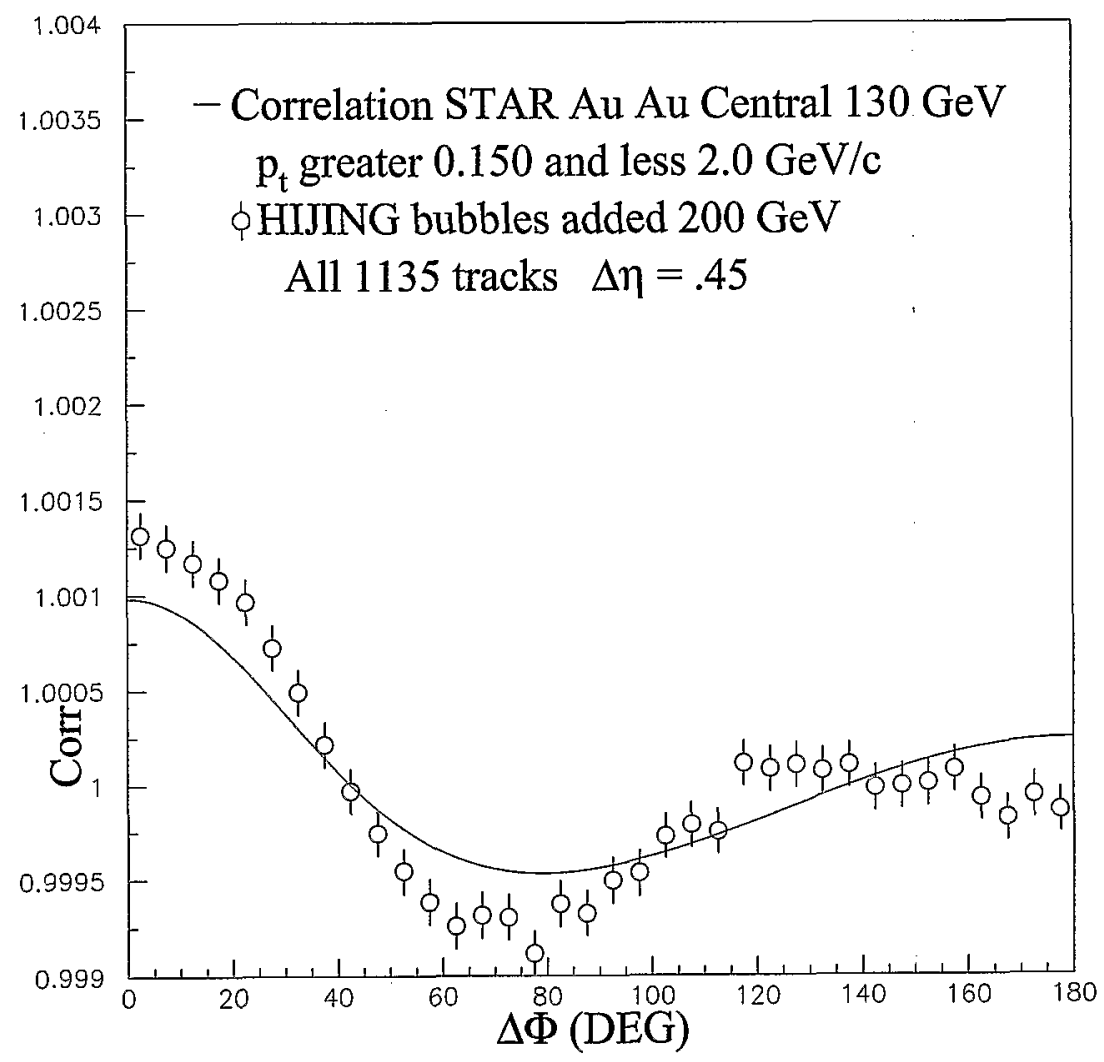

Figure 23: Comparison of the CI correlation in the $\Delta \eta=0.45$ bin of the bubble model prediction for $\sqrt{s_{N N}}=200 \mathrm{GeV}$ with experimental results at $\sqrt{s_{N N}}=130 \mathrm{GeV}$. See section VI of the text for approximations made and discussion. 


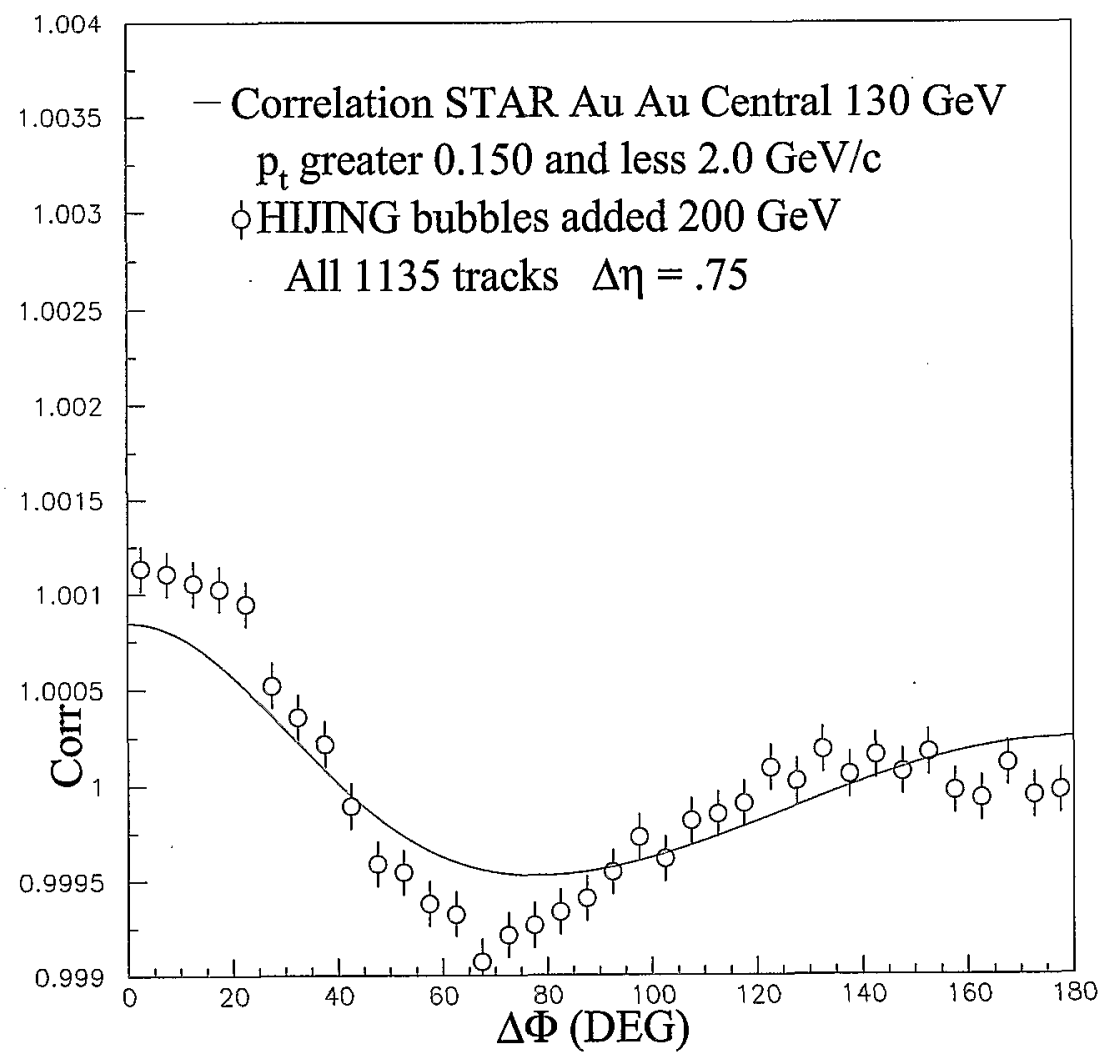

Figure 24: Comparison of the CI correlation in the $\Delta \eta=0.75$ bin of the bubble model prediction for $\sqrt{s_{N N}}=200 \mathrm{GeV}$ with experimental results at $\sqrt{s_{N N}}=130 \mathrm{GeV}$. See section VI of the text for approximations made and discussion. 


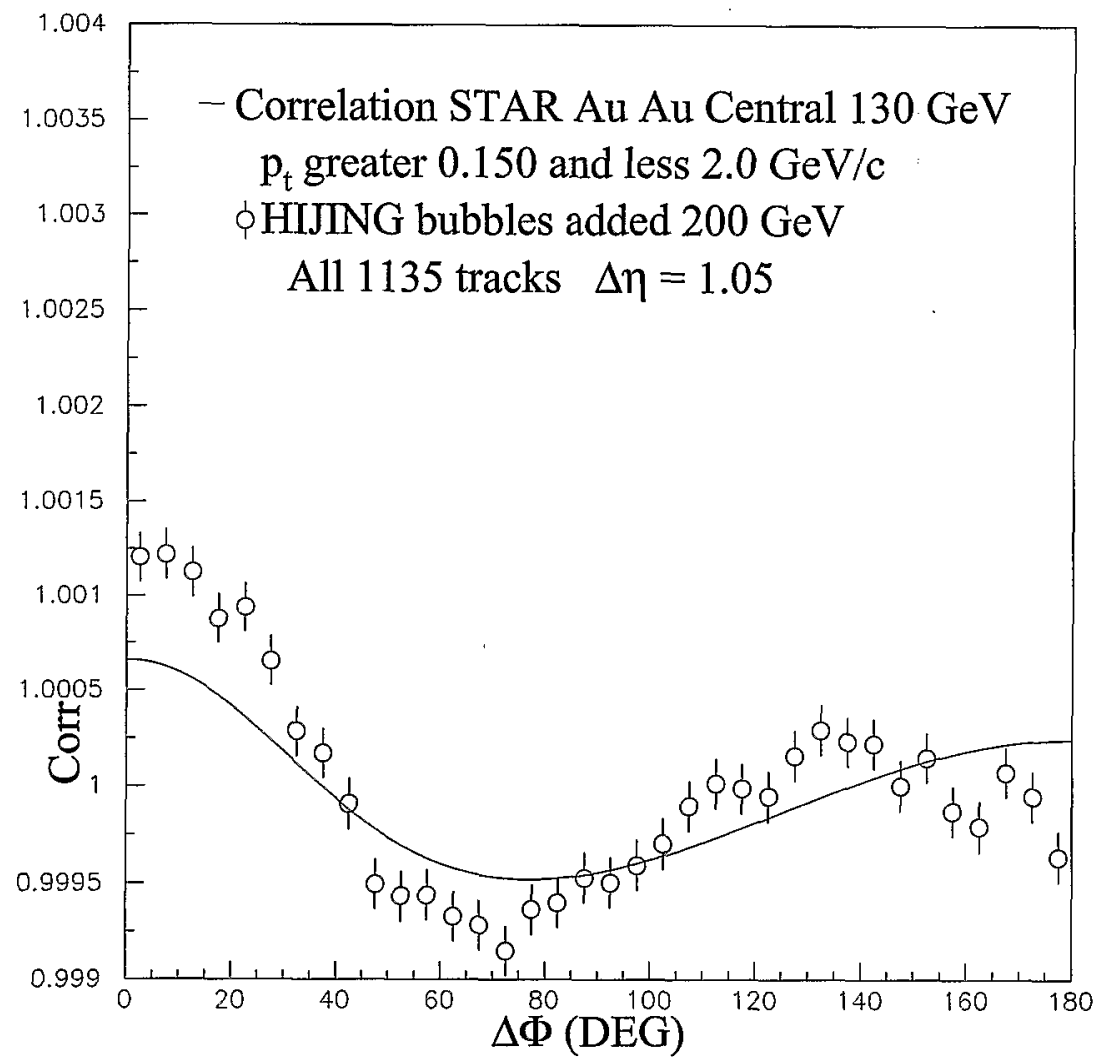

Figure 25: Comparison of the CI correlation in the $\Delta \eta=1.05$ bin of the bubble model prediction for $\sqrt{s_{N N}}=200 \mathrm{GeV}$ with experimental results at $\sqrt{s_{N N}}=130 \mathrm{GeV}$. See section VI of the text for approximations made and discussion. 


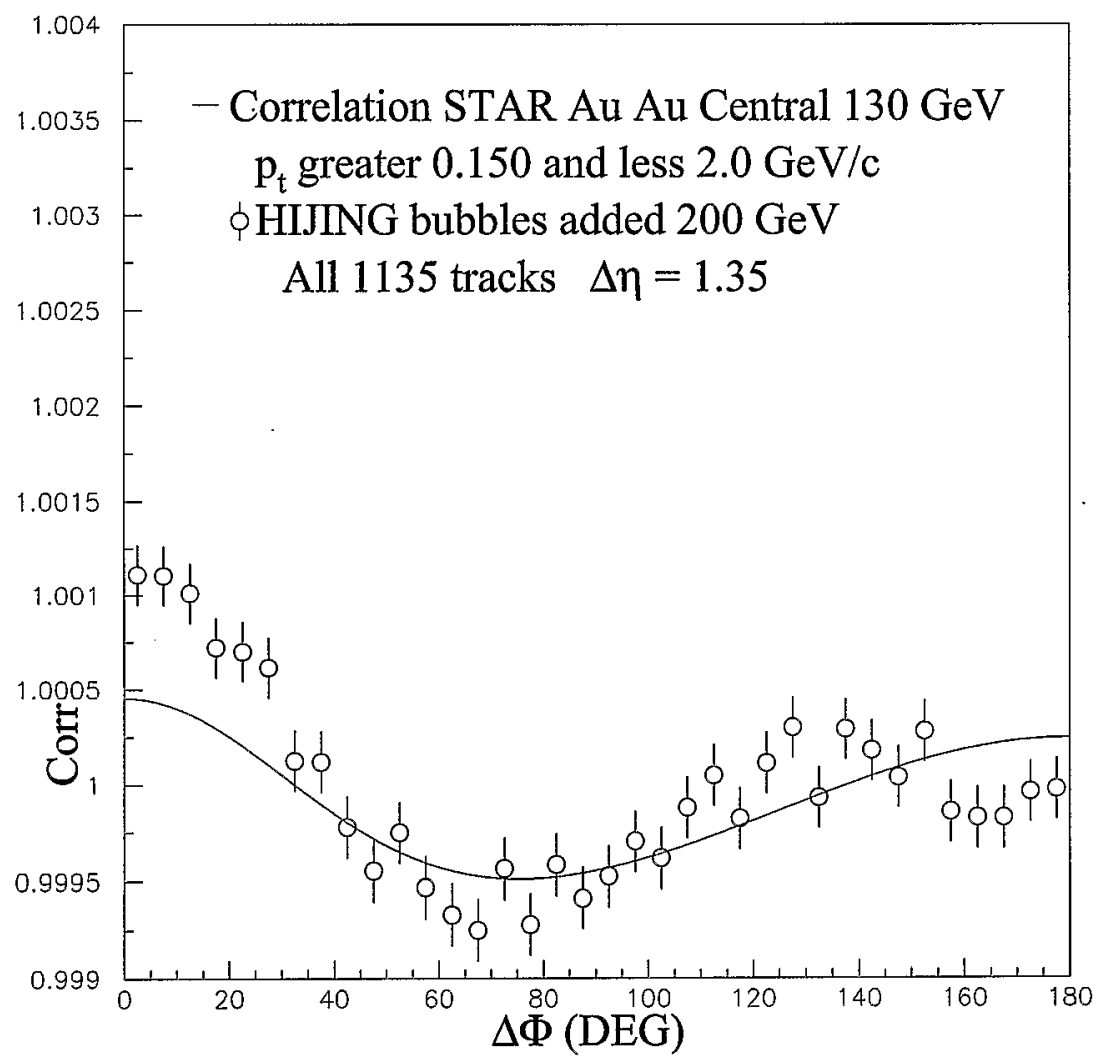

Figure 26: Comparison of the CI correlation in the $\Delta \eta=1.35$ bin of the bubble model prediction for $\sqrt{s_{N N}}=200 \mathrm{GeV}$ with experimental results at $\sqrt{s_{N N}}=130 \mathrm{GeV}$. See section VI of the text for approximations made and discussion. 
Note the qualitative similarity of the bubble model predictions and the correlation data fit it is compared with. Generally the correlation of the data as a function of $\Delta \phi$ is smaller for each $\Delta \eta$ bin particularly in the near forward angles where the bubble model contribution is largest. The above study seems to be consistent with surface emission from bubbles as expected from the bubble model with roughly, half of the charged particles at the lower $p_{t}$ being rescattered as they make their way out of the interior of the fireball. These ideas are consistent with jet quenching of ref.[5], and large energy loss if any parton tries to move across the interior region of the fireball. Thus the large angular correlations that are seen at RHIC[16] are explained as due to bubble hot spots on the surface of the fireball at freezeout.

\section{$7 \quad$ Summary and Discussion}

In this article we revisit our bubble model[1]. We expanded the model to successfully reach our goal of developing a parton inspired model for these bubbles and describe how the bubble of partons fragments into charged particles. With the help of RHIC data[14] we were able to tune the model and determine the number of partons (3-4 essentially all gluons) in a bubble, and their longitudinal momenta at a fixed phi. Experimental information[8] about how strong jet quenching is, allowed us to expand the upper limit of transverse momentun $\left(p_{t}\right)$ to $4.0 \mathrm{GeV} / \mathrm{c}$, which includes the quark-quark recombination region without including a significant jet contamination. Our earlier paper[1] chose to avoid the above $2.0 \mathrm{GeV} / \mathrm{c} p_{t}$ range in order to exclude significant jet effects. Utilizing more recently available data we have been able to make the approximation for central $\mathrm{Au}+\mathrm{Au}$ collisions that jets play a small part in our increased $0.8 \mathrm{GeV} / \mathrm{c}<p_{t}<4.0 \mathrm{GeV} / \mathrm{c}$ range, unless one triggers on high $p_{t}$ particles. Thus for our un-triggered particle correlations in the bubble model, jets can be ignored. In the longitudinal phase space of our fragmenting bubbles quark-quark recombination gives the same velocity to di-quarks thus twice the transverse momentum. This recombination increases the number of baryons and anti-baryons in the parton fragmentation of the bubble compared to the normal yield of jet fragmentation. In Figure 15 we show experimental data which agree with the bubble model prediction of the ratio of proton plus anti-proton to all charged particles as a function of $p_{t}$ (see Section 4).

In Section 6 and Figures 22 to 26, we compared the predictions of the bubble model with the correlation data analysis of $\Delta \Phi-\Delta \eta$ in ref.[16]. This experiment observed particles in the $p_{t}$ range 0.15 to $2.0 \mathrm{GeV} / \mathrm{c}$. Thus most of the particles had a lower $p_{t}$ than the $>0.8$ $\mathrm{GeV} / \mathrm{c}$ cut that we normally use in the bubble model. However we, were able to show that the large $\Delta \Phi-\Delta \eta$ correlations observed were in qualitative agreement with the emission of particles from the fireball surface bubbles as predicted by the bubble model.

We have made many quantitative predictions for two particle correlations that can be quantitatively tested by high precision data from RHIC which is expected to become available in the near future.

The geometry of the expanding fireball surface along with the HBT source size of about $2 \mathrm{fm}$ radius chosen for our adjoining bubbles, lead to 12 bubbles in an $8 \mathrm{fm}$ radius ring, perpendicular to and centered around the beam. The bubble ring is wrapped around the 
outer surface of the fireball at mid rapidity [1]. We have seen from Table I that each bubble on the average has $6 \mathrm{GeV} / \mathrm{c} p_{t}$ when we consider charged particles above $0.8 \mathrm{GeV} / \mathrm{c}$. We also found that when we consider soft particles from the bubble the charged particles account for $11 \mathrm{GeV} / \mathrm{c} p_{t}$. If we now consider the neutral contribution, we end up with around 16 $\mathrm{GeV} / \mathrm{c} p_{t}$ per bubble. We have shown above that each bubble on the average contains 27 $\mathrm{GeV}$ of energy in charged particles which increases to $40 \mathrm{GeV}$ when we consider the neutral particles. It is of interest to note that on the average each bubble contains approximately one thousandth the total initial energy of the $\mathrm{Au}+\mathrm{Au}$ system. Since we have 12 bubbles this implies that there is $480 \mathrm{GeV}$ or $\sim 500 \mathrm{GeV}$ of energy stored in the bubbles on the surface of the fireball.

Our first goal is to establish the existence of a bubble type substructure after comparison with high precision data, some of which is expected in the near future. The ultimate goal is to investigate the nature and content of the bubble structure and determine if it has final state properties implying a QGP.

\section{References}

[1] S.J. Lindenbaum, R.S. Longacre and M. Kramer, Eur. Phys. C 30, 241-253 (2003), DOI: 10. 1140/epjc/s2003-01 268-3.

[2] a) L. Schroeder and S.J. Lindenbaum, Large Magnetic Spectrometer. S.J. Lindenbaum part II Proc. RHIC Workshop: Experiments for Relativistic Heavy Ion Collider, April 15-19, 1985, eds. P.E.Haustein and C.L.Woody,pp. 211-252, Brookhaven National Laboratory, Upton, New York. b) S.J. Lindenbaum, An Approximately $4 \pi$ Tracking Magnetic Spectrometer for RHIC. Proc. of the Second Workshop on Experiments and Detectors for Relativistic Heavy Ion Collider(RHIC), May 25-29, 1987, eds. Hans Georg Ritter and Asher Shor, pp. 146-165, Lawrence Berkeley Laboratory, Berkeley, California. c) S.J. Lindenbaum, A $4 \pi$ Tracking Magnetic Spectrometer for RHIC. Proc.of the Third Workshop on Experiments and Detectors for Relativistic Heavy Ion Collider(RHIC), Brookhaven National Laboratory, July 11-22, 1988, eds. B. Sivakumar and P. Vincent, pp. 82-96, Brookhaven National Laboratory, Upton, NY. d) S.J. Lindenbaum, et al., A $4 \pi$ Tracking TPC Magnetic Spectrometer for RHIC. Proc. of the fourth Workshop on Experiments and Detectors for a Relativistic Heavy Ion Collider (RHIC), Brookhaven National Laboratory, July 2-7, 1990, eds. M. Fatyga and B. Moskowitz, pp. 169-206, Brookhaven National Laboratory, Upton, NY.

[3] L. Van Hove, Hadronization Quark-Gluon Plasma in Ultra-Relativistic Collisions CERN-TH (1984) 3924, Hadronization Model for Quark-Giuon Plasma in UltraRelativistic Collisions, Z. Phys. C27 (1985) 135.

[4] S.J. Lindenbaum and R.S. Longacre, J. Phys. G: 26 (2000) 937-956.

[5] J. Adams et al., Phys. Rev. C. 70 (2004) 054907.

[6] F. Karsch, Nucl. Phys. A 698 (2002) 199c. 
[7] J. Adams et al., Phys. Rev. C. 71 (2005) 044906, S.S. Adler et al., Phys Rev. Lett. 93 (2004) 1523092.

[8] C. Adler et al., Phys. Rev. Lett. 87 (2001) 112303, Phys. Rev. Lett. 92 (2004) 112301.

[9] J. Adams et al., Phys. Rev. Lett. 95 (2005) 152301.

[10] R.J. Fries,B. Muller,C. Nonaka and S.A. Bass, Phys. Rev. C. 68 (2003) 044902.

[11] X.N. Wang and M. Gyulassy, Phys. Rev. D 44 (1991) 3501.

[12] K.H. Ackerman et al., Phys. Rev. Lett. 86 (2001) 402, C. Adler et al., Phys. Rev. Lett. 90 (2003) 032301, J. Adams et al., Phys. Rev. C. 72 (2005) 014904.

[13] T. Sjostrand and M. van Zijil, Phys. Rev. D 36 (1987) 2019.

[14] R.S. Longacre for the Star Collaboration, Proceedings of the International Workshop on: Light Cone Physics: Hadron and Beyond, Insititute for Particle Physics Phenomenology, University of Durham, UK, Aug 5th-9th,2003,editor:S. Dalley, 110-117.

[15] S.S. Adler et al., Phys Rev. Lett. 91 (2003) 172301.

[16] J. Adams et al., nucl-ex/0411003. 